\title{
LIFTING THE VEIL ON OBSCURED ACCRETION: ACTIVE GALACTIC NUCLEI NUMBER COUNTS AND SURVEY STRATEGIES FOR IMAGING HARD X-RAY MISSIONS
}

\author{
D. R. Ballantyne ${ }^{1}$, A. R. Draper ${ }^{1}$, K. K. Madsen ${ }^{2}$, J. R. Rigby ${ }^{3,6,7}$, and E. Treister ${ }^{4,5,8}$ \\ ${ }^{1}$ Center for Relativistic Astrophysics, School of Physics, Georgia Institute of Technology, Atlanta, GA 30332, USA; david.ballantyne@ physics.gatech.edu \\ ${ }^{2}$ Space Radiation Laboratory, California Institute of Technology, MC: 220-47, Pasadena, CA 91125, USA \\ ${ }^{3}$ Carnegie Observatories, Carnegie Institution of Washington, Pasadena, CA 91101, USA \\ ${ }^{4}$ Institute for Astronomy, University of Hawaii, Honolulu, HI 96822, USA \\ ${ }^{5}$ Departamento de Astronomia, Universidad de Concepcion, Casilla 160-C, Concepcion, Chile \\ Received 2010 November 29; accepted 2011 May 4; published 2011 July 6
}

\begin{abstract}
Finding and characterizing the population of active galactic nuclei (AGNs) that produces the X-ray background (XRB) is necessary to connect the history of accretion to observations of galaxy evolution at longer wavelengths. The year 2012 will see the deployment of the first hard X-ray imaging telescope which, through deep extragalactic surveys, will be able to measure the AGN population at the energies where the XRB peaks $(\sim 20-30 \mathrm{keV})$. Here, we present predictions of AGN number counts in three hard X-ray bandpasses: 6-10 keV, $10-30 \mathrm{keV}$, and 30-60 keV. Separate predictions are presented for the number counts of Compton thick AGNs, the most heavily obscured active galaxies. The number counts are calculated for five different models of the XRB that differ in the assumed hard X-ray luminosity function, the evolution of the Compton thick AGNs, and the underlying AGN spectral model. The majority of the hard X-ray number counts will be Compton thin AGNs, but there is a greater than tenfold increase in the Compton thick number counts from the $6-10 \mathrm{keV}$ to the $10-30 \mathrm{keV}$ band. The Compton thick population shows enough variation that a hard X-ray number counts measurement will constrain the models. The computed number counts are used to consider various survey strategies for the NUSTAR mission, assuming a total exposure time of 6.2 Ms. We find that multiple surveys will allow a measurement of Compton thick evolution. The predictions presented here should be useful for all future imaging hard X-ray missions.
\end{abstract}

Key words: galaxies: active - galaxies: nuclei - galaxies: Seyfert - surveys - X-rays: diffuse background - X-rays: galaxies

Online-only material: color figures

\section{INTRODUCTION}

The vast majority of the growth of supermassive black holes (SMBHs) is driven by accretion (e.g., Marconi et al. 2004; Merloni \& Heinz 2008; Di Matteo et al. 2008). Therefore, a complete census of accreting SMBHs throughout cosmic time is necessary to quantify the efficiency of accretion (e.g., Sołtan 1982; Yu \& Tremaine 2002; Shankar et al. 2004; Yu \& Lu 2008), which, in turn, elucidates the connection between black hole growth and galaxy evolution (e.g., Haiman et al. 2004, 2007; Hopkins \& Hernquist 2009; Shankar et al. 2009). All efficiently accreting SMBHs are intrinsically luminous X-ray sources, while the underlying host galaxy is, in general, a much fainter X-ray emitter. The cosmic X-ray background (XRB) is then naturally interpreted as being the integral emission of all accreting SMBHs in the universe, and the hard slope of the XRB spectrum indicates that most of the active galactic nuclei (AGNs) are obscured behind substantial columns of gas and dust in the host galaxy (e.g., Setti \& Woltjer 1989; Comastri et al. 1995; Ueda et al. 2003; Gilli et al. 2001, 2007). This expectation was spectacularly confirmed by the numerous deep surveys performed by Chandra and XMM-Newton in the $2-10 \mathrm{keV}$ band that resolved $>80 \%$ of the XRB into individual objects (e.g., Brandt \& Hasinger 2005; Worsley et al. 2005). Multiwavelength follow-up observations of these sources show that a large fraction of them are obscured, type 2, AGNs

\footnotetext{
6 Carnegie Fellow.

7 Current address: NASA Goddard Space Flight Center, Code 665, Greenbelt, MD 20771, USA.

8 Chandra/Einstein Fellow.
}

at redshifts of $z \sim 1$ (Alexander et al. 2001; Tozzi et al. 2001; Barger et al. 2002, 2005; Stern et al. 2002; Brusa et al. 2010).

However, summing the emission from all the AGNs detected by Chandra and other missions working below $10 \mathrm{keV}$ cannot account for the observed peak of the XRB at $\sim 30 \mathrm{keV}$ (e.g., Gilli et al. 2007). The missing AGNs are likely dominated by Compton thick sources, those behind obscuring column densities $N_{\mathrm{H}} \gtrsim \sigma_{\mathrm{T}}^{-1} \approx 10^{24} \mathrm{~cm}^{-2}$. The combination of photoelectric absorption and Compton scattering reduces the observed flux of these AGNs to such a degree that, if they lie at any reasonable cosmological distance, they are invisible even in the deepest Chandra or XMM-Newton surveys (Brandt \& Hasinger 2005). There are currently three strategies that are being employed to discover and identify Compton thick AGNs. The first is to look for the "waste heat" being re-radiated by the absorber in the mid-infrared. Several groups have uncovered a population of Compton thick candidates at $z \gtrsim 1$ using this idea (Stern et al. 2005; Alonso-Herrero et al. 2006; Polletta et al. 2006; Daddi et al. 2007; Alexander et al. 2008; Fiore et al. 2008, 2009), but the constraints are complicated by the necessity to separate out contributions from obscured star formation (e.g., Mushotzky 2004; Donley et al. 2008; Eckart et al. 2010; Georgakakis et al. 2010). The second method to detect Compton thick AGNs is to search at hard X-ray energies $(\gtrsim 20 \mathrm{keV})$ where the sources are less affected by obscuration (although still suffer from Compton scattering losses). The hard X-ray detectors on board BeppoSAX, Swift, and INTEGRAL have been able to reveal local (i.e., $z \approx 0$ ) Compton thick AGNs through this method (e.g., Bassani et al. 1999; Vignali \& Comastri 2002; Malizia et al. 
2009a). Recent surveys by the latter two missions have measured the fraction of all AGNs that are Compton thick at $z \approx 0$ to be $\sim 10 \%-20 \%$, depending on the luminosity range considered and the details of the source selection (Sazonov et al. 2007; Tueller et al. 2008; Malizia et al. 2009b; Treister et al. 2009; Winter et al. 2009; Burlon et al. 2011). Finally, Compton thick AGNs may also be identified in pointed X-ray observations by searching for objects with specific optical characteristics (Gilli et al. 2011), or those that have very large $\mathrm{Fe} \mathrm{K} \alpha$ equivalent widths (e.g., Levenson et al. 2006; LaMassa et al. 2009; Comastri et al. 2011; Feruglio et al. 2011). However, these observations require very long exposure times and the translation from $\mathrm{Fe} \mathrm{K} \alpha$ line measurement to column density depends on model-dependent details such as the geometry and metallicity of the absorber (e.g., Murphy \& Yaqoob 2009).

The hard X-ray instruments flown in previous missions have had fairly poor sensitivity when compared to detectors at lower energies. Therefore, there is very little known about the Compton thick AGN population at $z>0$. Intriguingly, if one takes the infrared estimates of Compton thick densities at high redshift at face value, then there must be strong evolution of the Compton thick population (Draper \& Ballantyne 2010; Treister et al. 2010). This evolution might indicate that "Compton thickness" is an unique phase of AGN evolution and is tied to specific Eddington ratios or merger events (Draper \& Ballantyne 2010; Treister et al. 2010). Therefore, discovering the missing Compton thick AGNs beyond the local universe and tracing their evolution is vital to understanding a host of problems related to black hole fueling and galaxy evolution.

In 2012 the Nuclear Spectroscopic Telescope Array ${ }^{9}$ (NUSTAR; Harrison et al. 2010) will be launched as part of NASA's Small Explorer Program. This mission will be the first ever focusing hard X-ray telescope covering an energy range of 6-80 keV. It will therefore be imaging the sky at the energies where the XRB peaks and the Compton thick sources are most visible. One of the primary objectives of the baseline two-year NuSTAR mission is to perform extragalactic surveys to describe the AGN population and XRB emission at these energies. Other hard X-ray imaging missions, such as Astro-H (Takahashi et al. 2010; launching in 2013/2014) and the proposed New Hard $X$-ray Mission (Tagliaferri et al. 2010), will follow later this decade. The data produced by these missions will provide crucial tests of models of AGN and galaxy evolution.

Thus, in light of the upcoming launch of $N u S T A R$, there is a need for models of AGN evolution, and, specifically, Compton thick evolution, that can be used to compare against future hard $\mathrm{X}$-ray data. In addition, model predictions can also be used to assist the planning of the hard X-ray extragalactic surveys to ensure that a reasonable number of Compton thick AGNs are detected at several different redshifts. To be the most effective, several models, spanning a range of parameters, should be available to compare against the survey data with the goal that many of them will be eliminated. As a first step, this paper predicts the hard X-ray number counts for three different measurements of the AGN hard X-ray luminosity function (HXLF) with each requiring a different Compton thick fraction. In addition, we also predict counts for a model where the Compton thick fraction is a function of AGN Eddington ratio, and therefore varies with redshift and AGN luminosity. Finally, we also present a conservative model that predicts the smallest fraction of Compton thick AGNs consistent with the available

\footnotetext{
9 http://www.nustar.caltech.edu/
}

data. Our results are specifically designed for comparison with the upcoming hard X-ray surveys and are the first to show the effects of the different model parameters on the AGN and Compton thick counts.

The next section describes the various XRB models used to calculate the Compton thick and total AGN number counts. The counts are presented in Section 3. Section 4 then applies these results to the design of deep hard X-ray surveys, with a specific example for $N u S T A R$. Our conclusions for the NuSTAR mission and for other future hard X-ray missions are summarized in Section 5. This paper assumes the following cosmological parameters: $H_{0}=70 \mathrm{~km} \mathrm{~s}^{-1} \mathrm{Mpc}^{-1}, \Omega_{\Lambda}=0.7$, and $\Omega_{m}=0.3$.

\section{CALCULATION OF PREDICTED NUMBER COUNTS}

The integrated AGN number counts, $N(>S)$, in a specific energy band are

$$
\begin{aligned}
N(>S)= & \frac{c}{H_{0}} \int_{z_{\min }}^{z_{\max }} \int_{\max \left(\log L_{X}(\min ), \log L_{X}\left(\mathrm{~S}_{\mathrm{N}_{\mathrm{H}}}\right)\right)}^{\log L_{X}(\max )} \frac{d \Phi\left(L_{X}, z\right)}{d \log L_{X}} \\
& \times \frac{d_{l}^{2}}{(1+z)^{2}\left(\Omega_{m}(1+z)^{3}+\Omega_{\Lambda}\right)^{1 / 2}} d \log L_{X} d z,
\end{aligned}
$$

where $d \Phi\left(L_{X}, z\right) / d \log L_{X}$ is a given HXLF, $d_{l}$ is the luminosity distance to redshift $z$, and $L_{X}\left(S_{N_{\mathrm{H}}}\right)$ is the unabsorbed rest-frame 2-10 keV luminosity that gives an observed frame flux $S_{N_{\mathrm{H}}}$ for a source at $z$ averaged over the $N_{\mathrm{H}}$ distribution for that $L_{X}$ and $z$. The integrals are evaluated from $z_{\min }=0$ to $z_{\max }=5$, and $\log \left(L_{X}(\min ) / \mathrm{erg} \mathrm{s}^{-1}\right)=41.5$ to $\log \left(L_{X}(\max ) / \mathrm{erg} \mathrm{s}^{-1}\right)=48$. The integrated counts are computed in three energy bands: ${ }^{10}$ 6-10 keV, 10-30 keV, and 30-60 keV. As described below, the calculation of the expected number counts relies on a number of quantities which are poorly constrained or subject to significant uncertainties.

\subsection{Unabsorbed AGN Spectrum}

The conversion between luminosity and flux requires a spectral model for the average AGN population at a given $L_{X}$ and $z$. The assumed unabsorbed rest-frame AGN spectrum is comprised of a power law with photon index $\Gamma$ that varies from source to source (e.g., Winter et al. 2009), an exponential cutoff at $E_{\text {cut }}=250 \mathrm{keV}$, and a reflection component. The distribution of the power-law cutoff energies is not observationally constrained and will be an important measurement for the upcoming hard X-ray missions. However, Gilli et al. (2007) find that including a range of cutoff energies in their XRB synthesis model has a negligible effect on the results; therefore, we do not consider this parameter to be a significant source of uncertainty in the predictions.

The reflection component hardens the spectrum above $\gtrsim 10 \mathrm{keV}$ and produces an $\mathrm{Fe} \mathrm{K} \alpha$ line. A reflection spectrum may arise from either the accretion disk or from cold, dense gas at large distances from the black hole (e.g., Ross \& Fabian 2005; Murphy \& Yaqoob 2009). Thus, the strength of the reflection component in an AGN spectrum may vary significantly from source to source, although it is known that, on average, it cannot be very large (Gandhi et al. 2007; Ballantyne 2010). In addition, there is observational evidence that the strength of the reflection spectrum from the distant reprocessor is anti-correlated with the luminosity (Bianchi et al. 2007; Shu et al. 2010).

\footnotetext{
10 To assist comparisons with previous observations and models, the Appendix shows predictions in the 5-10 keV band.
} 


\section{2. $N_{\mathrm{H}}$ Distribution and Compton Thick AGNs}

The basic spectral model will suffer some obscuration that depends on the fraction of obscured AGNs at a specific $L_{X}$ and $z$, as well as the distribution of obscuring column densities. The fraction of absorbed, or type 2, AGNs $\left(f_{2}\right)$ is defined as those objects observed through column densities $N_{\mathrm{H}} \geqslant 10^{22} \mathrm{~cm}^{-2}$. This fraction is observed to decrease with AGN luminosity (e.g., Ueda et al. 2003; Barger et al. 2005; La Franca et al. 2005; Tueller et al. 2008) and, tentatively, increase with $z$ (La Franca et al. 2005; Ballantyne et al. 2006; Treister \& Urry 2006; Hasinger 2008). The distribution of column densities that comprise the obscured and unobscured population has been measured in the local universe or in a redshift and luminosity integrated sense (Comastri et al. 1995; Risaliti et al. 1999; Ueda et al. 2003; Dwelly et al. 2005; Tozzi et al. 2006). However, it is possible (perhaps likely) that the distribution of column densities will also depend on $L_{X}$ and $z$.

Previous deep X-ray surveys by Chandra and XMM-Newton are not sensitive to most Compton thick AGNs; therefore the space density and evolution of these objects represents the largest uncertainty in the census of AGN. Additional unobserved objects have to be added to the HXLF in order to boost the integrated emission to fit the XRB spectrum, and/or the measured $z=0$ Compton thick space density (Draper \& Ballantyne 2009). As mentioned above, Compton thick AGNs are obscured by columns $N_{\mathrm{H}} \gtrsim 10^{24} \mathrm{~cm}^{-2}$, but once $N_{\mathrm{H}}$ reaches a few times $10^{24} \mathrm{~cm}^{-2}$ the transmitted continuum becomes severely suppressed (Matt et al. 1999). Indeed, extreme Compton thick AGNs with $N_{\mathrm{H}} \gtrsim 10^{25} \mathrm{~cm}^{-2}$ will be, for all practical purposes, invisible even in the deepest hard X-ray surveys. Consequently, such objects cannot provide a significant contribution to the XRB, and this paper defines Compton thick AGNs as those with columns $24 \leqslant \log N_{\mathrm{H}} \leqslant 25$.

The simplest method for including Compton thick AGNs into an XRB model is as a straightforward extension of the Compton thin population that evolve with other obscured AGNs (i.e., along with $f_{2}$ ). This is quantified by the Compton thick fraction, $f_{\mathrm{CT}}$, defined as the fraction of all type 2 AGNs that are Compton thick. For example, $f_{\mathrm{CT}}=0.5$ means that there are as many Compton thick AGNs as Compton thin type $2 \mathrm{~s}$.

\subsection{X-Ray Luminosity Functions}

In recent years there have been several measurements of the HXLF that have significantly different predictions for the value and redshift evolution of the faint end slope of the HXLF (Ueda et al. 2003; Barger et al. 2005; La Franca et al. 2005; Silverman et al. 2008; Ebrero et al. 2009; Yencho et al. 2009; Aird et al. 2010). For a given spectral model and $N_{\mathrm{H}}$ distribution, these differences in the HXLF can result in factors of 2-3 variations in the number density of Compton thick AGNs (Draper \& Ballantyne 2009).

\subsection{Model Definitions}

Given all the uncertainties associated with the calculation of number counts, we consider five separate models to determine the impact of our ignorance of many of the above parameters. The most interesting physical parameters that will likely be constrained by the hard X-ray number counts are the HXLF and Compton thick fraction. Therefore, we consider three XRB models with a fixed Compton thick fraction that differ only in the assumed HXLF. The fourth XRB model uses one of the test HXLFs (specifically, the Ueda et al. 2003 HXLF), but assumes a more complicated form for the Compton thick evolution, where the Compton thick fraction depends on the Eddington ratio. The fifth and final model provides a lower limit to the number of Compton thick AGNs. This model uses the Ueda et al. (2003) HXLF and a fixed Compton thick fraction, but assumes a brighter AGN spectrum and the minimum allowable Compton thick density. Thus, this model predicts the most conservative view of the Compton thick population that is consistent with the available data. ${ }^{11}$

\subsubsection{HXLF Variable Models}

In the following models, the reflection spectrum is calculated using the "reflion" model (Ross \& Fabian 2005) assuming a solar Fe abundance. This reflection spectrum is averaged over all viewing angles and is added to the power law such that the equivalent width of the narrow $\mathrm{Fe} \mathrm{K} \alpha$ line agrees with the observed X-ray Baldwin effect (Bianchi et al. 2007). The corresponding reflection fraction drops from $\sim 1$ at low luminosities to close to zero at high luminosities. To account for the observed distribution of photon indices, both the power law and the reflection spectrum are Gaussian averaged about $\Gamma=1.9$ with $\Gamma_{\min }=1.5, \Gamma_{\max }=2.3$, and $\sigma_{\Gamma}=0.2$ (e.g., Gilli et al. 2007).

The fraction of obscured AGNs, $f_{2}$, is assumed to be dependent on both redshift and $L_{X}$, such that $f_{2} \propto(1+z)^{a}\left(\log L_{X}\right)^{-b}$ where $a=0.4^{12}$ (Ballantyne et al. 2006; Treister \& Urry 2006) and $b=4.7$. The normalization of $f_{2}$ is set by fixing the type 2 to type 1 ratio at $4: 1$ for $z=0$ and $\log \left(L_{X} / \mathrm{erg} \mathrm{s}^{-1}\right)=41.5$, as determined from observations (Maiolino \& Rieke 1995) and XRB modeling (Ballantyne et al. 2006; Gilli et al. 2007). The redshift evolution is halted at $z=1$ corresponding to the flattening evolution of the cosmic star formation rate density (e.g., Hopkins \& Beacom 2006). The unabsorbed type 1 sources are evenly distributed over the columns $\log N_{\mathrm{H}}=20,20.5,21$, and 21.5, while Compton thin type 2 sources are evenly distributed over the columns $\log N_{\mathrm{H}}=22,22.5,23$, and 23.5.

The Compton thick fraction, $f_{\mathrm{CT}}$, is determined by requiring the Compton thick AGN space density at $z \approx 0$ with $\log \left(L_{X} / \mathrm{erg} \mathrm{s}^{-1}\right)>43$ to be $\approx 3.6 \times 10^{-6} \mathrm{Mpc}^{-3}$, in agreement with the absorption corrected local space density measurement from Swift/BAT and INTEGRAL (Treister et al. 2009; Rigby et al. 2009; Draper \& Ballantyne 2010). This value is very similar to the density predicted by the Gilli et al. (2007) model (e.g., Alexander et al. 2008), which, judging from Figure 3 in the paper by Treister et al. (2009), suggests that $\sim 15 \%$ of $z \sim 0$ AGNs in this luminosity range are Compton thick. The Compton thick AGNs are evenly distributed over the columns $\log N_{\mathrm{H}}=24,24.5$, and 25, consistent with the limits from local observations (Treister et al. 2009), and the Compton thick absorption profiles are taken from the Monte Carlo models of Matt et al. (1999). A scattered reflection spectrum with a luminosity of $2 \%$ of the intrinsic luminosity is used in place of the transmitted absorption spectrum for the $\log N_{\mathrm{H}}=25$ spectral model, and is added to the $\log N_{\mathrm{H}}=24.5$ spectrum (e.g., Gilli et al. 2007).

\footnotetext{
11 Interested readers can perform a similar test by computing the number counts for the Gilli et al. (2007) XRB synthesis model using their webtool at http://www.bo.astro.it/ gilli/counts.html. Note that the Compton thick fraction of this model is similar to the HXLF variable models used here. As the Appendix shows, the Gilli et al. (2007) predictions are within the range of those studied in this paper, and do not change any of our conclusions. 12 If $a=0.62$ and continues out to $z=2$ (Hasinger 2008), then the $10-30 \mathrm{keV}$ Compton thick counts are increased by $\lesssim 13 \%$ at $1<z<2$, but are reduced by $\lesssim 9 \%$ at $z<0.5$.
} 
The three HXLFs used in these models (in order of increasing Compton thick fraction) are the ones published by Ueda et al. (2003; $f_{\mathrm{CT}}=0.3$, blue lines in all figures), La Franca et al. (2005; $f_{\mathrm{CT}}=0.4$, green lines), and Aird et al. (2010; $f_{\mathrm{CT}}=0.5$, red lines). The Ueda et al. (2003) and La Franca et al. (2005) HXLFs are best modeled by luminosity dependent density evolution, while Aird et al. (2010) find that luminosity and density evolution (LADE) is the best description of their data.

\subsubsection{Compton Thick Evolution Model}

In addition to the above three models, we also consider the scenario where the Compton thick fraction $\left(f_{\mathrm{CT}}\right)$ evolves separately from the less obscured AGN. Specifically, we make use of the composite model of Draper \& Ballantyne (2010) who assumed that $f_{\mathrm{CT}}$ was a function of the AGN Eddington ratio. After considering several scenarios and comparing to the high- $z$, IR-derived Compton thick space densities, Draper \& Ballantyne (2010) estimated that Compton thick AGNs make up $\sim 86 \%$ of all AGNs with Eddington ratios greater than 0.9, $\sim 60 \%$ of AGNs with Eddington ratios less than 0.01, and $0 \%$ of AGNs at intermediate Eddington ratios. This model makes use of the Ueda et al. (2003) HXLF and recovers the observed $z=0$ Compton thick density (Draper \& Ballantyne 2010). All other parameters are identical to the previously described models. This model is shown as the black line in all figures.

\subsubsection{The Conservative Compton Thick Model}

We define a model, described by Treister et al. (2009), that provides a lower limit to the Compton thick population that is consistent with all available data. This model assumes a constant power law with $\Gamma=1.9$ and a cutoff energy of $300 \mathrm{keV}$, attenuated by photoelectric absorption and a constant Compton reflection component as given by Magdziarz \& Zdziarski (1995). The reflecting material is assumed to provide a reflection fraction of 1.0, has twice the solar iron abundance, and has an average inclination angle to the line of sight of $45^{\circ}$. The larger iron abundance and constant reflection fraction result in a slighter more luminous AGN spectrum above $10 \mathrm{keV}$ when compared to the models described in Sections 2.4.1 and 2.4.2.

The $N_{\mathrm{H}}$ distribution employed here is the one shown in Figure 2 of Treister et al. (2009) and was originally derived from a torus geometry corresponding to an AGN unification model with an aspect ratio matching the local ratio of Type 2 to Type 1 Seyfert galaxies (Treister et al. 2004). However, it is fully consistent with the observed distributions in various hard X-ray surveys (Comastri et al. 1995; Risaliti et al. 1999; Ueda et al. 2003; Dwelly et al. 2005; Tozzi et al. 2006). In this $N_{\mathrm{H}}$ distribution the majority of the Compton thick AGNs have $N_{\mathrm{H}}<$ $10^{24.5} \mathrm{~cm}^{-2}$, which results in a more luminous average Compton thick spectrum than used in the models described above. Since this paper is focused on either the Compton thick AGNs or on the total AGN population, the remaining differences in the two $N_{\mathrm{H}}$ distributions have no impact on the results. The luminosity dependence of the obscured AGN fraction $f_{2}$ falls linearly from $f_{2}=1$ to 0 between $L_{X}=10^{42}$ and $3 \times 10^{46} \mathrm{erg} \mathrm{s}^{-1}$ (Treister $\&$ Urry 2005). The redshift dependence of $f_{2}$ is the same as the other models, and the Ueda et al. (2003) HXLF is assumed.

The Compton thick fraction of this model is set to be consistent with the observed fraction of AGNs at $z \approx 0$ that are Compton thick, as deduced from hard X-ray surveys by Swift and INTEGRAL (Sazonov et al. 2007; Tueller et al. 2008; Treister et al. 2009). This corresponds to a fraction of all $z \sim 0$ AGNs that are Compton thick of $\sim 10 \%$ (Treister et al. 2009;

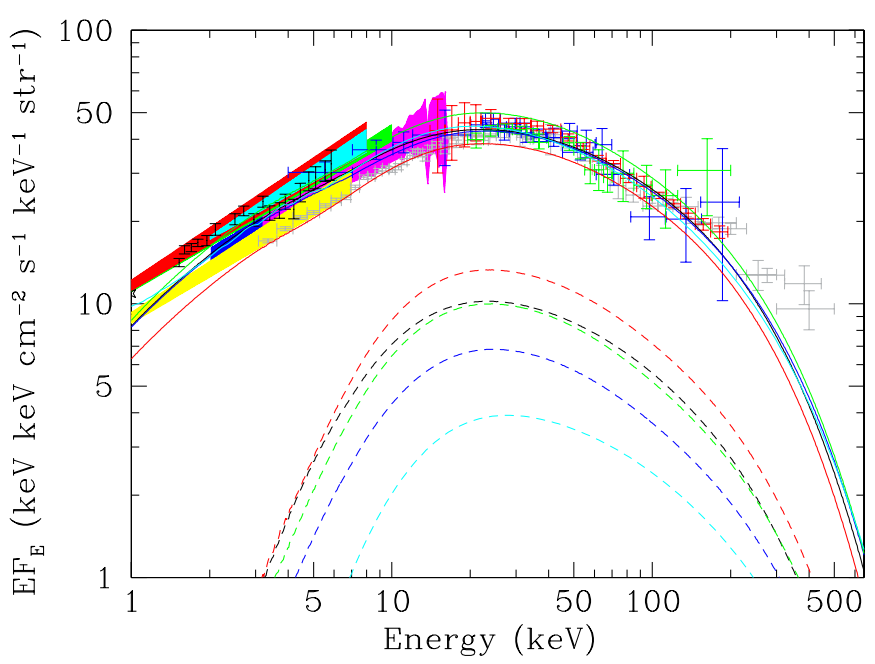

Figure 1. Solid lines show the X-ray background spectra predicted by the five AGN evolution models considered here: Ueda et al. (2003) HXLF (blue), La Franca et al. (2005) HXLF (green), Aird et al. (2010) HXLF (red), the Draper \& Ballantyne (2010) composite model (black), and the conservative Compton thick model (Treister et al. 2009) (cyan). The dashed lines plot the Compton thick contribution to the X-ray background. The X-ray background data are from the following instruments: blue: Advanced Satellite for Cosmology and Astrophysics (ASCA) GIS (Kushino et al. 2002); magenta: Rossi X-ray Timing Explorer (Revnivtsev et al. 2003); green: XMM-Newton (Lumb et al. 2002); red: BeppoSAX (Vecchi et al. 1999); yellow: ASCA SIS (Gendreau et al. 1995); cyan: XMM-Newton (De Luca \& Molendi 2004); gray data: HEAO-1 (Gruber et al. 1999); blue data: INTEGRAL (Churazov et al. 2007); red data: Swift/BAT (Ajello et al. 2008); black data: Swift/XRT (Moretti et al. 2009); green data: INTEGRAL (Türler et al. 2010).

(A color version of this figure is available in the online journal.)

Figure 3), and is likely to be a strict lower limit due to the observational biases of those surveys. The extinction corrected density used to normalize the Compton thick fraction in the models described in Section 2.4.1 attempts to partially address those biases (Rigby et al. 2009). Thus, this model presents the most conservative view of the Compton thick population and is shown as the cyan line in all plots.

\subsection{Summary of Models}

Predictions of the hard X-ray number counts rely on several parameters or distributions that are only partially observationally constrained. Thus, five separate models were defined to fully explore the sensitivity of the results to these assumptions. Of the five, three explored differences in the measured AGN HXLF (Section 2.4.1), one considered a model where the Compton thick fraction was a function of AGN accretion rate (Section 2.4.2), and one provides the most conservative view of the Compton thick population (Section 2.4.3). Figure 1 shows the XRB spectrum, as well as the contribution from Compton thick AGNs for these five models. All models provide an adequate fit to the XRB spectrum, which nicely illustrates the degeneracy of synthesis modeling. The redshift evolution of the $\log \left(L_{X} / \mathrm{erg} \mathrm{s}^{-1}\right)>43$ Compton thick space density predicted by the models is shown in Figure 2. The differences in the three different HXLFs are easily observed with the Compton thick density increasing with the necessary $f_{\mathrm{CT}}$. At $z \lesssim 1$, the Ueda et al. (2003) HXLF requires the least amount of Compton thick AGNs and the Aird et al. (2010) HXLF has the most. Above $z \gtrsim 1$, the different evolutions of the HXLFs become apparent and the La Franca et al. (2005) and Aird et al. (2010) HXLFs predict a similar Compton thick density. The brighter spectral 


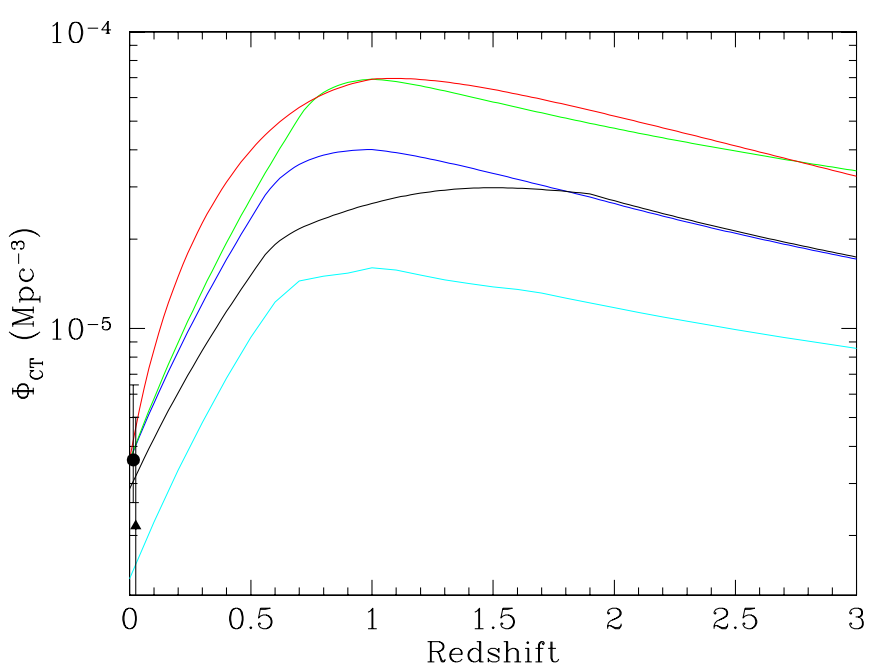

Figure 2. Predicted space density of Compton thick AGNs with $\log \left(L_{X} / \mathrm{erg} \mathrm{s}^{-1}\right)>43$ as a function of $z$. The different models are distinguished by the line color: Ueda et al. (2003) HXLF (blue), La Franca et al. (2005) HXLF (green), Aird et al. (2010) HXLF (red), the Draper \& Ballantyne (2010) composite model (black), and the conservative Compton thick model (Treister et al. 2009) (cyan). The triangle denotes the local Compton thick density calculated by Treister et al. (2009), while the circle shows the same density after correcting for the flux-luminosity relation reported by Rigby et al. (2009).

(A color version of this figure is available in the online journal.)

model and lower Compton thick fraction employed in the Treister et al. (2009) model result in a low Compton thick density at all $z$ 's. Finally, the evolving Compton thick model predicts a small fraction of high luminosity Compton thick AGNs at $z<1$ because there are very few high Eddington ratio AGNs to host such obscured objects. Thus, there is a significant difference in the predicted densities of Compton thick objects at $z<1$, implying that deep hard X-ray surveys may be capable to distinguish among these models.

\section{RESULTS}

\subsection{Number Counts}

Figures 3-5 show the predicted $N(>S)$ distributions for multiple redshift ranges in the 6-10 keV, $10-30 \mathrm{keV}$, and 30-60 keV bands, respectively. The solid lines plot the counts for all AGN, while the dashed lines present the predictions for Compton thick sources. The colors differentiate between the various HXLFs and Compton thick evolutions: La Franca et al. (2005) HXLF (green), Aird et al. (2010) HXLF (red), the composite model of Draper \& Ballantyne (2010) (black), and the Treister et al. (2009) model (cyan). For clarity, the model based on the Ueda et al. (2003) HXLF has been omitted from the plots. Recall that both the Draper \& Ballantyne (2010) and the Treister et al. (2009) make use of the Ueda et al. (2003) HXLF.

\subsubsection{All AGNs}

Concentrating initially on the counts of all AGNs (solid lines), we see that the numbers are very similar to those observed in the softer energy bands by Chandra and XMM-Newton (e.g., Rosati et al. 2002; Lehmer et al. 2005; Mateos et al. 2008). This is not surprising: most AGNs are only moderately obscured and so would appear in both $2-8 \mathrm{keV}$ and $10-30 \mathrm{keV}$ surveys. These results imply that most of the sources found in hard X-ray surveys will have Chandra and/or XMM-Newton counterparts

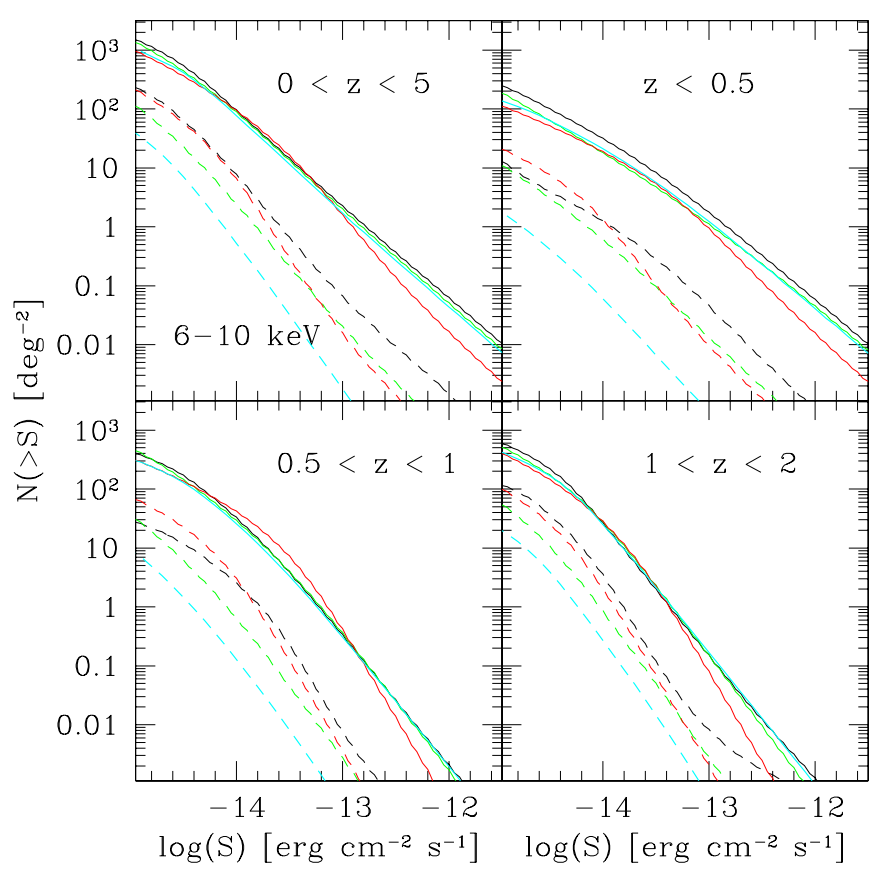

Figure 3. Solid lines plot the integrated number counts of all AGNs in the 6-10 keV energy band. The contribution to the counts by Compton thick AGNs are shown as dashed lines. The top-left panel plots the counts over the redshift range $z=0-5$. The other panels show the integrated counts over smaller ranges of redshift. The colors differentiate between the various HXLFs and/or Compton thick evolutions assumed by the model: La Franca et al. (2005) HXLF (green), Aird et al. (2010) HXLF (red), the Draper \& Ballantyne (2010) composite model (black), and the conservative Compton thick model (Treister et al. 2009) (cyan). For clarity the model that uses the Ueda et al. (2003) HXLF has not been plotted. (A color version of this figure is available in the online journal.)

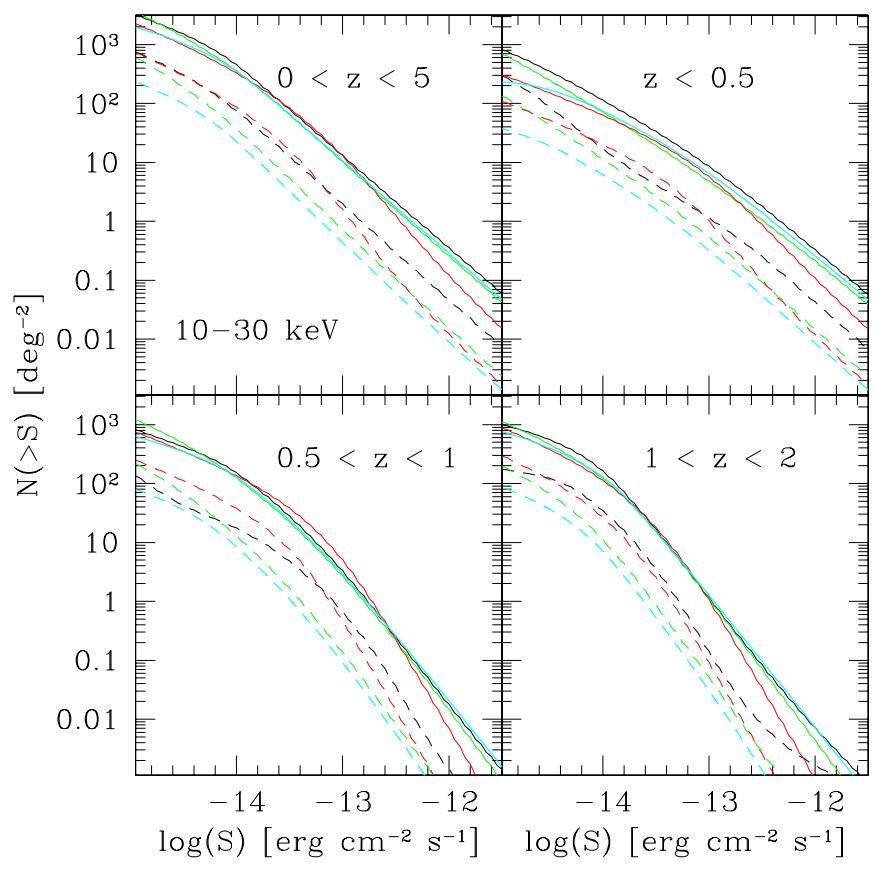

Figure 4. Same as Figure 3, but for the 10-30 keV energy band. (A color version of this figure is available in the online journal.)

with similar fluxes, especially for AGNs at higher $z$ where the $K$-correction has moved the absorption out of the Chandra or XMM-Newton band (see Section 3.2).

The Ueda et al. (2003) and La Franca et al. (2005) HXLF predict very similar numbers for the total counts, except for 


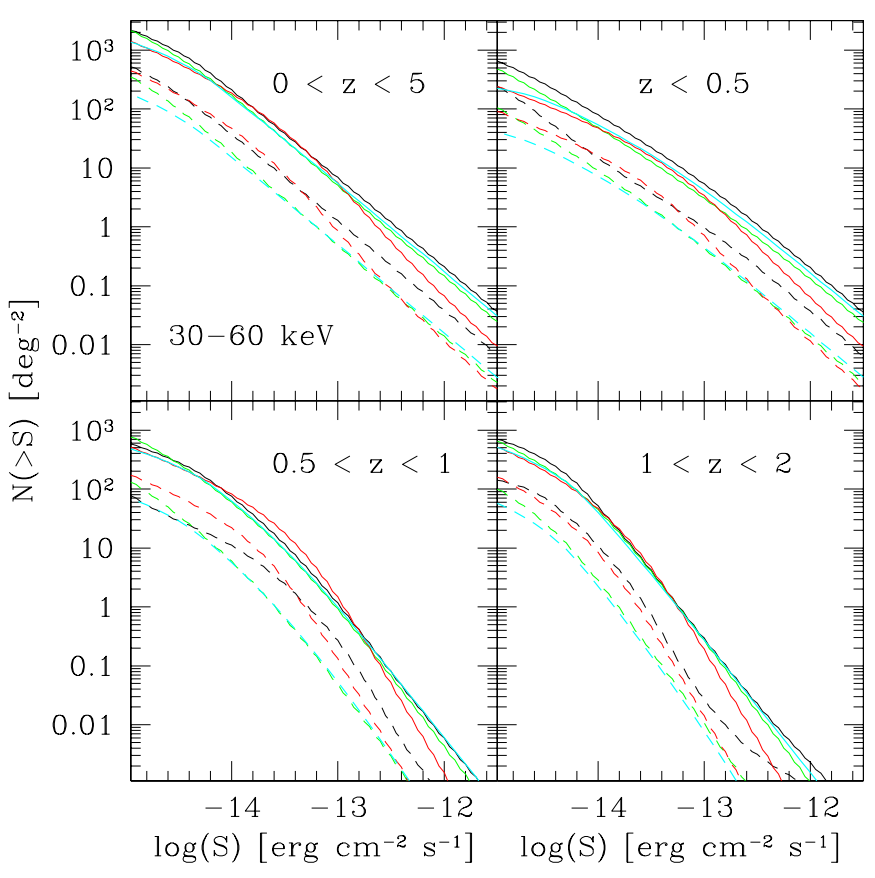

Figure 5. Same as Figure 3, but for the 30-60 keV energy band. (A color version of this figure is available in the online journal.)

$z>2$, where there are very few constraining data points. In contrast, the Aird et al. (2010) HXLF consistently predicts smaller values for the counts, especially at fluxes greater than $10^{-13} \mathrm{erg} \mathrm{cm}^{-2} \mathrm{~s}^{-1}$. This result is because the Aird et al. (2010) HXLF predicts fewer high luminosity AGNs at all redshifts. Moreover, the LADE model does not require an increase in the space density of low- $z$, low-luminosity AGNs. The predicted number counts from the model that uses the Aird et al. (2010) HXLF show a significant enough difference from the other models that surveys will be able to test the viability of this HXLF at different redshift ranges.

\subsubsection{Compton Thick AGNs}

Turning now to the Compton thick objects, we see that the number counts are uniformly small, comprising only 5\%-10\% of the total AGN counts. Therefore, discovering and measuring the evolution of these objects will be a tough observational challenge. However, Figures 3-5 immediately show the advantage of hard X-ray imaging to find Compton thick AGNs. At all redshifts, the number counts of Compton thick source increase by factors of $\sim 10-100$ when moving from the $6-10 \mathrm{keV}$ to the 10-30 keV band. This result emphasizes that hard X-ray imaging is an important method to identify significant numbers of Compton thick candidates. Interestingly, there is little difference in the expected Compton thick number counts between the $10-30 \mathrm{keV}$ and $30-60 \mathrm{keV}$ bands. In fact, the counts drop slightly in the $30-60 \mathrm{keV}$ band at $z \gtrsim 1$ as the $K$-correction causes the observed frame flux to drop. This is a result of the high-energy cutoff in the AGN power law and electron recoil in the reflection spectrum, both of which reduce the intensity of the total spectrum at high energies. We conclude that, in order to maximize the sensitivity to Compton thick AGNs, high energy observations need only to extend out to $30-40 \mathrm{keV}$ (depending on the redshift of interest).

The combination of the different HXLFs and assumptions of the Compton thick evolution result in wide range of predictions for the number counts of these AGNs. Figures 3-5 indicate that the best discrimination of the models will result from objects at $z<1$. In particular, the model of Draper \& Ballantyne (2010), where Compton thick objects are comprised of a combination of low Eddington ratio objects and very high Eddington ratio AGNs (black lines), predicts a significantly different number count distribution than the other four models where Compton thick objects are an unchanging fraction of the AGN population, and evolve in the same way as the less obscured sources. For example, at $z<0.5$ the Draper \& Ballantyne (2010) model predicts $\sim 2 \times$ more Compton thick AGNs with a $10-30 \mathrm{keV}$ flux larger than $10^{-13} \mathrm{erg} \mathrm{cm}^{-2} \mathrm{~s}^{-1}$ than the La Franca et al. (2005) HXLF (Figure 4). In the Draper \& Ballantyne (2010) model, these high flux, low- $z$ Compton thick AGNs are dominated by low accretion rate objects similar to the Circinus galaxy. Pushing to higher redshifts moves a survey closer to the quasar era where high Eddington ratio accretion is more common and the Draper \& Ballantyne (2010) model predicts additional Compton thick sources. The factor between the evolving and fixed Eddington ratio models at a depth of $10^{-13} \mathrm{erg} \mathrm{cm}^{-2} \mathrm{~s}^{-1}$ is therefore boosted to $\sim 5$ in the $0.5<z<1$ bin. In addition, the evolving Compton thick model has a shallower slope at bright fluxes than the fixed Compton thick fraction models due to low luminosity, weakly accreting Compton thick AGNs. Thus, the Draper \& Ballantyne (2010) model can be easily tested with a Compton thick AGN number counts measurement that moves beyond $z \sim 0.1$ and to a 10-30 keV depth of $10^{-13} \mathrm{erg} \mathrm{cm}^{-2} \mathrm{~s}^{-1}$. In this way hard X-ray surveys will be able to measure the evolution of Compton thick AGNs and determine their relation, if any, to stages of galaxy evolution. Indeed, confirming that the Compton thick AGN fraction is larger at higher redshift will have implications for understanding the physics of AGN triggering and the AGN-galaxy connection. Finally, as seen by comparing the conservative Treister et al. (2009) model (cyan lines) to the other predictions, the number of Compton thick AGNs detected by hard X-ray surveys will help test the assumptions made in that model, in particular the shape of the average spectral model and the value of the local Compton thick density.

\subsection{Identification and Optical Detectability of the Hard X-Ray Sources}

As seen above, hard X-ray imaging is extremely efficient at detecting AGNs, but additional analysis will be required in order to begin to use these detections to investigate problems in galaxy and black hole evolution. This is particularly true for the Compton thick AGNs, as they cannot be identified from hard X-ray imaging alone-additional data is necessary to determine the level of obscuration for each hard X-ray source. Moreover, understanding the nature of the detected hard X-ray sources will require that the host galaxies be sufficiently bright so that redshifts and stellar population measurements can be made at optical and infrared wavelengths. Both of these problems can be addressed if the hard X-ray surveys are performed on fields with significant multiwavelength coverage. For example, we have seen that the majority of hard X-ray sources should have Chandra or XMM-Newton counterparts provided that at least moderately deep (i.e., a flux limit of $\sim 10^{-16}$ to $10^{-15} \mathrm{erg} \mathrm{cm}^{-2} \mathrm{~s}^{-1}$ ) soft X-ray observations exist in the observed field(s). Figure 6 shows the relationship between the soft and hard X-ray fluxes for the spectral model described in Section 2.4.1. The left panel plots $0.5-10 \mathrm{keV}$ fluxes for AGNs with (intrinsic, unabsorbed) $L_{X}=10^{43} \mathrm{erg} \mathrm{s}^{-1}$ against the 10-30 keV flux for different obscuring column densities. As an example of the typical Chandra sensitivity, the horizontal 

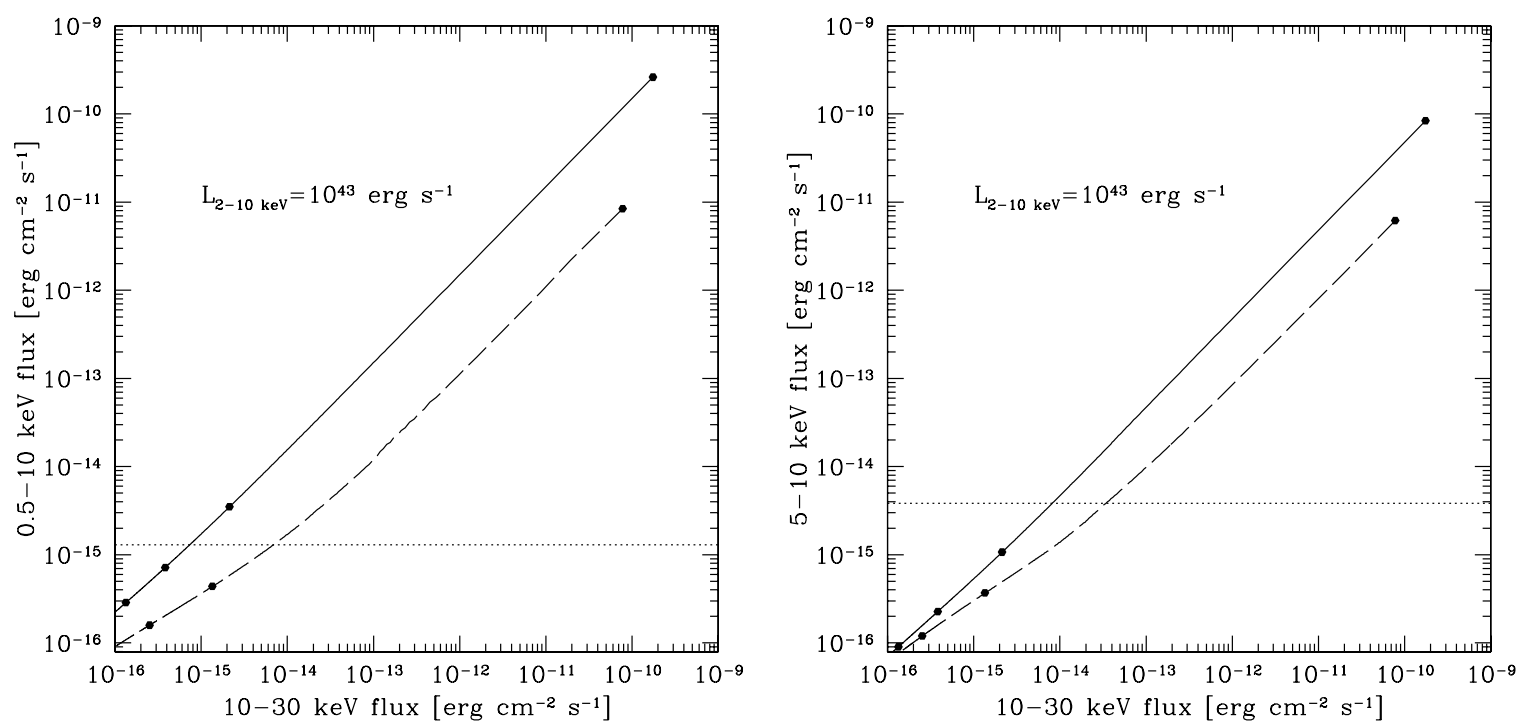

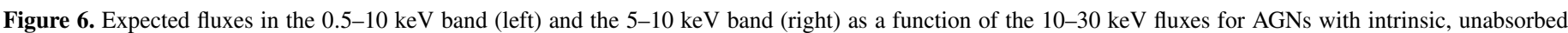

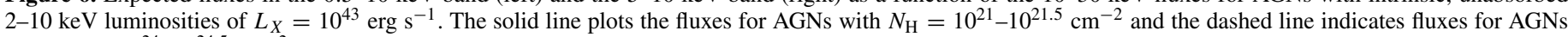

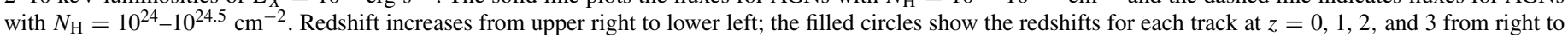

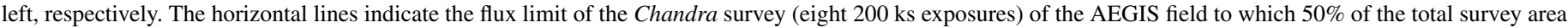
is complete (Laird et al. 2009).

line shows the $50 \%$ completeness flux limit from the Chandra observations of the All-wavelength Extended Groth Strip International Survey (AEGIS, eight 200 ks exposures; Laird et al. 2009). The solid line shows the fluxes for unabsorbed AGNs, and, for all realistic flux limits reached by the next generation of hard X-ray surveys $\left(\sim 10^{-14}\right.$ to $10^{-13} \mathrm{erg} \mathrm{cm}^{-2} \mathrm{~s}^{-1}$ in the $10-30 \mathrm{keV}$ band), these sources will have $0.5-10 \mathrm{keV}$ fluxes greater than the flux limit for moderate/deep Chandra observations in many survey regions. Thus, $\sim 90 \%$ of the hard X-ray detections will have soft X-ray counterparts that can be combined with the hard X-ray data to estimate $N_{\mathrm{H}}$.

The dashed line plots the fluxes for Compton thick AGNs, and the effects of absorption are clearly seen in the predicted 0.5-10 keV fluxes. Although Compton thick AGNs will be an order of magnitude brighter in the 10-30 keV band, ones detected in the hard X-rays still seem to be at or above the detectability limit of the deepest Chandra surveys (if they lie at low $z$ ). However, this plot neglects the steep decline in the ACIS-I effective area at energies greater than $5 \mathrm{keV}$. This effect is illustrated in the right panel of Figure 6 which plots the 5-10 keV flux versus the 10-30 keV flux. This figure clearly shows that some Compton thick AGNs will be detectable in hard X-ray surveys, but beneath the sensitivity limit of deep Chandra surveys. These results again indicate the power of hard X-ray imaging, with no decline in effective area above $5 \mathrm{keV}$, to discover the most heavily obscured AGNs between $z=0$ and 1 . Thus, in addition to those that might be found by combining Chandra and hard X-ray detections, Compton thick candidates can also be identified by searching for objects that have no 5-10 keV Chandra counterparts. Analysis using infrared data (e.g., Alexander et al. 2008) can then be pursued on these candidates to confirm the identification. Finally, there may be an unknown number of heavily obscured objects that have extremely weak scattered components in the soft band (e.g., Ueda et al. 2007). Such objects would also be detected out to moderate redshift by hard X-ray imaging.

Redshifts are typically obtained from spectroscopic or photometric measurements of the host galaxy. Therefore, it is impor- tant to consider the expected $R$-band magnitudes for different $10-30 \mathrm{keV}$ fluxes to obtain a sense of the difficulty of identifying the host galaxy of the hard X-ray detected AGNs. This is done by using the empirical relations between X-ray luminosity and absolute $R$-band magnitude reported by La Franca et al. (2005), measured independently for obscured and unobscured AGNs:

$$
\log L_{R}=(0.959 \pm 0.025) \log L_{X}+(2.2 \pm 1.1),
$$

for unobscured AGNs and

$$
\log L_{R}=(0.462 \pm 0.026) \log L_{X}+(23.7 \pm 1.1)
$$

for obscured ones, where $L_{X}$ is the intrinsic luminosity in the rest-frame $2-10 \mathrm{keV}$ band. These equations are obviously an approximation, as they do not take into account some of the more detailed properties of the host galaxies, like evolving stellar populations or varying amounts of extinction. ${ }^{13}$ Such considerations are particularly important for obscured and Compton thick AGNs, in which the optical light is dominated by the host galaxy. The results are indistinguishable from the calculations, based on a dusty torus model, by Treister et al. (2004), which assumed the same host galaxy for all AGNs.

For the deepest hard X-ray surveys with a limiting $10-30 \mathrm{keV}$ sensitivity of $10^{-14} \mathrm{erg} \mathrm{cm}^{-2} \mathrm{~s}^{-1}$, most AGNs will have bright optical magnitudes, $R<23$ (AB), and, thus for surveys in well studied fields, the vast majority of the sources will have detections at other wavelengths and measured redshifts. For a very shallow X-ray survey $\left(\sim 10^{-13} \mathrm{erg} \mathrm{cm}^{-2} \mathrm{~s}^{-1}\right.$ depth), most AGNs will be brighter than $R \sim 20$ mag (AB), and hence within reach of the Sloan Digital Sky Survey.

\section{SURVEY STRATEGIES FOR HARD X-RAY MISSIONS}

\subsection{Area versus Depth}

Deep extragalactic surveys require significant investments of observing time and so need to be carefully planned in order to

\footnotetext{
13 However, the La Franca et al. (2005) sample has a similar $z$ distribution as will be found in the next generation of hard X-ray surveys, so the observed evolution of $R$-band luminosity should be similar.
} 

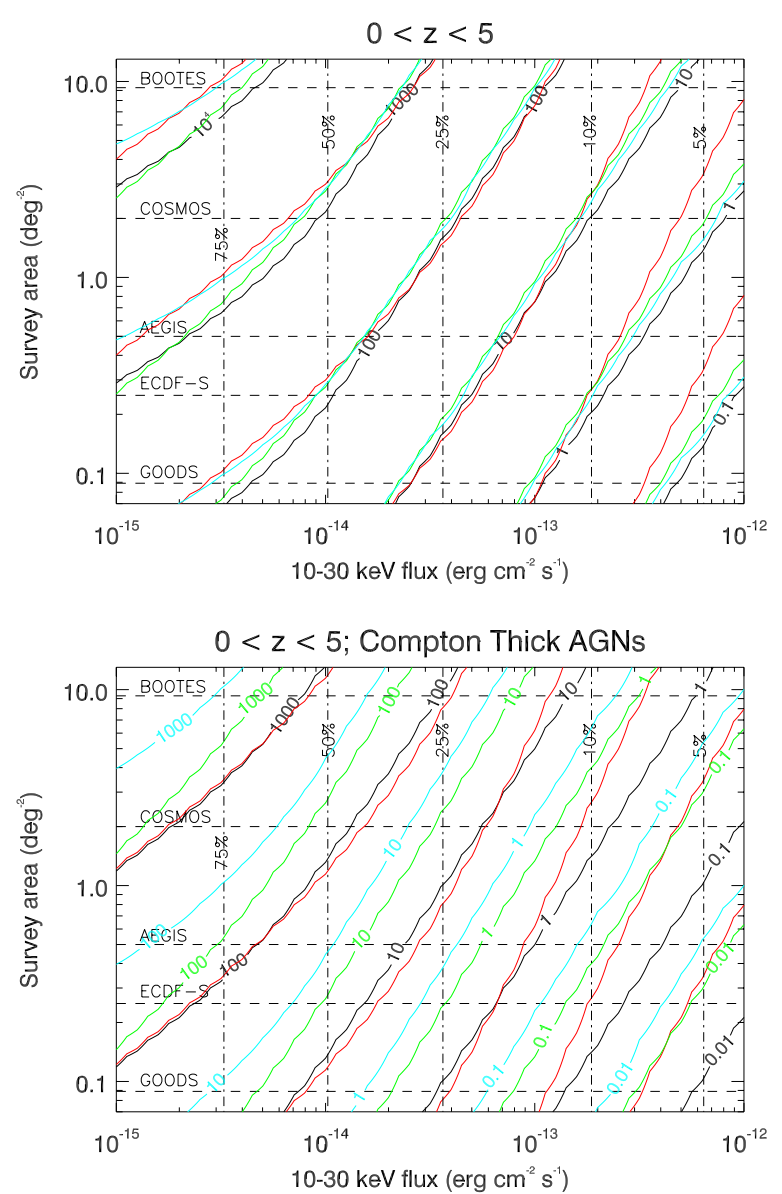

Figure 7. Top: contours of number of AGNs as a function of survey area and $10-30 \mathrm{keV}$ sensitivity. Results for the models are plotted using the same color scheme as previous figures with the Ueda et al. (2003) HXLF omitted for clarity. These contours include all sources in the range $0<z \leqslant 5$. The contour levels increase by factors of 10 from 0.1 sources (lower-right corner of the figure) to 10,000 sources (upper-left corner of the figure). Only the black contours are labeled. The horizontal lines show the areas covered by some well known multiwavelength surveys. The vertical lines indicate the flux level required to reach a certain percentage of the X-ray background (as judged by the Ueda et al. 2003 model) in the 10-30 keV band. Bottom: contours of Compton thick AGNs. The contour levels increase by factors of 10 from 0.01 sources (lowerright corner of the figure) to 1000 sources (upper-left corner of the figure).

(A color version of this figure is available in the online journal.)

maximize the scientific return. One important consideration in survey planning is the tradeoff between depth and area, as the luminosities and redshifts of sources detected in a very deep and narrow field are often very different from those uncovered in a shallower but wider field. The predicted number counts shown in Figures 3-5 contain all the information necessary for survey planning, but require additional manipulation to see the effects of surveys of differing areas. Therefore, Figures 7 and 8 present examples of a new way of plotting the predicted AGN and Compton thick AGN counts that is specifically designed for planning hard X-ray surveys. Each figure plots contours of numbers of AGNs (upper panel for all AGNs; lower panel for Compton thick AGNs) as a function of survey area and $10-30 \mathrm{keV}$ flux. The different colors indicate the predictions for four of the five different models considered in this paper (see Section 2.4). The dashed horizontal lines in each plot show the area of five regions with good multiwavelength coverage: Boötes Deep Wide-Field Survey (9.3 $\mathrm{deg}^{2}$; Murray et al. 2005), COSMOS (2 $\mathrm{deg}^{2}$; Hasinger et al. 2007; Elvis et al. 2009),
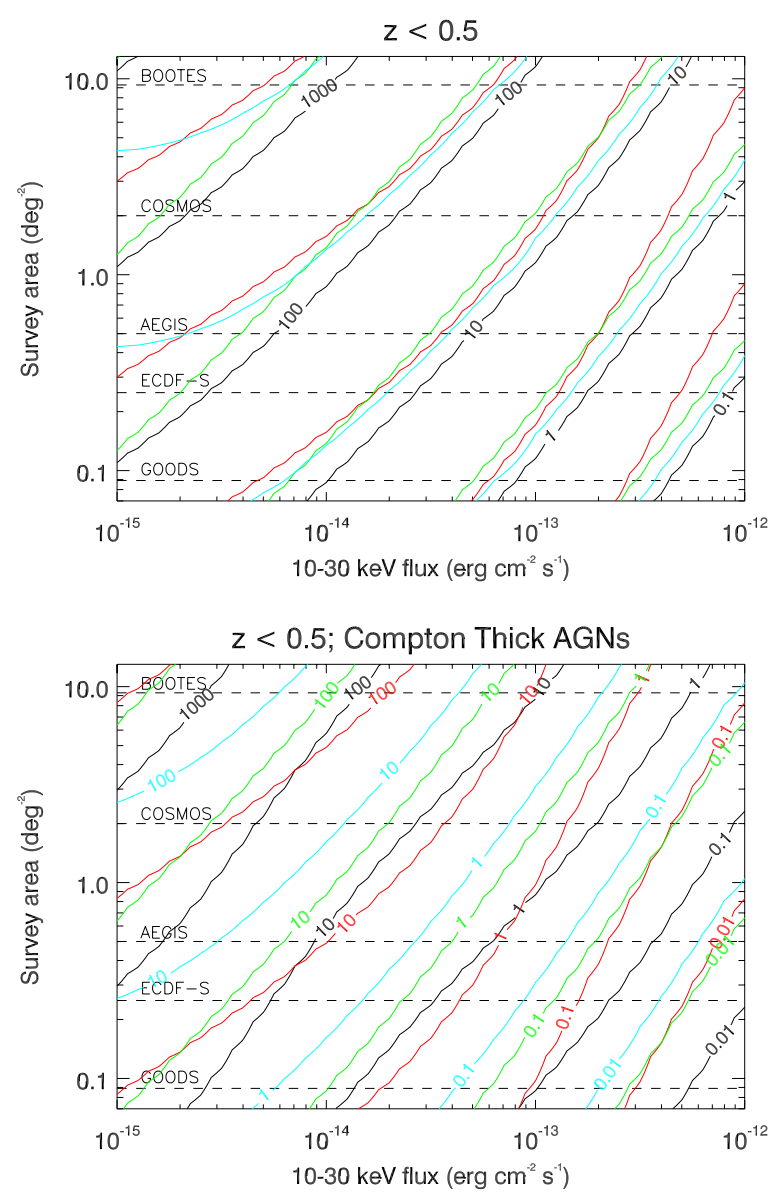

Figure 8. Same as Figure 7, however these contours include only those sources in the range $0<z<0.5$.

(A color version of this figure is available in the online journal.)

AEGIS (0.5 deg²; Davis et al. 2007; Laird et al. 2009), the Extended Chandra Deep Field South (ECDF-S, $0.25 \mathrm{deg}^{2}$; Lehmer et al. 2005), and the Great Observatories Origins Deep Survey (GOODS, $0.089 \mathrm{deg}^{2}$; Alexander et al. 2003; Luo et al. 2008). Finally, both panels of Figure 7 also have vertical lines indicating the flux needed (estimated using the Ueda et al. 2003 model) to reach a specific percentage of the integrated 10-30 keV XRB spectrum.

These figures contain a large amount of information and should be useful guides for planning future hard X-ray surveys. ${ }^{14}$ Close examination of the contours shows that, with the exception of the Aird et al. (2010) HXLF, all the models predict very similar numbers for the total number of AGNs. There is a slight spreading out of the contours at small fluxes, illustrating the uncertainty in the slope and evolution of the faint end of the HXLF. In contrast, there is a significant difference in the number of Compton thick AGNs predicted at a given flux and survey area, indicating that deep surveys will be able to easily distinguish between the various models.

Identifying highly embedded AGNs over a range of redshifts will be one of the principle goals of any hard X-ray survey; however, these AGNs will be faint and will require a significant investment of observing time to obtain a sizable number of sources. Figure 8 shows that an area the size of the COSMOS field must be surveyed to a depth of $(2-3) \times 10^{-14} \mathrm{erg} \mathrm{cm}^{-2} \mathrm{~s}^{-1}$

\footnotetext{
14 Plots for different energy bands and redshift ranges are available by contacting the authors.
} 
in the $10-30 \mathrm{keV}$ band to uncover at most 10 Compton thick AGNs at $z<0.5$. Similar numbers of Compton thick sources would also be found for that depth and sensitivity at $0.5<z<1$ and $1<z<2$. In contrast, a narrow field (such as the GOODS field) would have to be observed to a flux limit $10 \times$ fainter to obtain similar numbers of $z>0.5$ Compton thick AGNs. This survey, however, would not detect as many $z<0.5$ objects.

\subsection{Application to NUSTAR}

As mentioned in Section 1, only a portion of NuSTAR's baseline two-year mission will be dedicated to extragalactic surveys. The limited amount of time that is available requires that careful pre-launch planning be performed to optimize the survey strategy. Here we make use of the hard X-ray number counts presented above to predict the results of a NuSTAR survey of five different regions with significant ancillary multiwavelength data, including deep low-energy X-ray observations: Boötes, COSMOS, AEGIS, ECDF-S, and GOODS. Each survey has a total exposure time of $6.2 \mathrm{Ms}$, which, assuming $50 \%$ efficiency, would take 6 months to complete.

The NuSTAR sensitivities were derived using the "nustar sens" program provided by the NUSTAR instrument team. The program incorporates the $N u S T A R$ effective area, corrected for aperture stop, scattering from surface roughness, figure error, detector efficiency, and all known attenuation along the optical path in the form of thermal covers and beryllium windows. Combined with the GEANT4 (Agostinelli et al. 2003) simulated internal background and the diffuse XRB (for a low Earth orbit inclined at $5^{\circ}$ ), the program calculates a sensitivity map for the entire detector given a specific signal to noise, point-spread function (PSF), and input spectrum. A $\Gamma=1.7$ spectrum, appropriate for a moderately obscured AGN uncorrected for absorption and reflection (e.g., Malizia et al. 2003; Winter et al. 2009) is assumed for all calculations, and we require a $4 \sigma$ detection. Since the code was run for long exposures the signal to noise is in the Gaussian regime. The PSF used for the calculations follows a Gaussian+King profile and has a halfpower diameter (HPD) of 41". Because extraction from a region larger than the HPD will add more background than source counts, the HPD is used as the extraction region.

By definition, surveys require mapping areas greater than the detector field-of-view $\left(13^{\prime} \times 13^{\prime}\right)$, so survey sensitivities were calculated for two tiling strategies. The first, called "corner shift," is when the survey region is covered by non-overlapping fields of view with additional observations at the corners between fields of view. This strategy has the advantage that individual exposures can be fairly long, but there is a significant variation in sensitivity over the survey region as $N u S T A R$, like many other high-energy instruments, has an effective area that is a strong function of off-axis angle. The second strategy is called "half shift," and was calculated by shifting the detector sensitivity map over by half a field of view in both directions. Each individual pointing is shallower than the corner shift, but the half shift provides a more uniform sensitivity map over the survey region. One by-product of the half shift survey is that, due to the fact that the pointings are calculated such that they entirely cover the given survey size with the best sensitivity, the actual survey size ends up being larger than the input, with the extraneous area having lower sensitivity.

The area versus sensitivity curves for the five surveys are shown in Figure 9 with the corner shift surveys plotted as solid lines and the half shift surveys shown as dashed lines. The details of each survey-including the number of pointings,

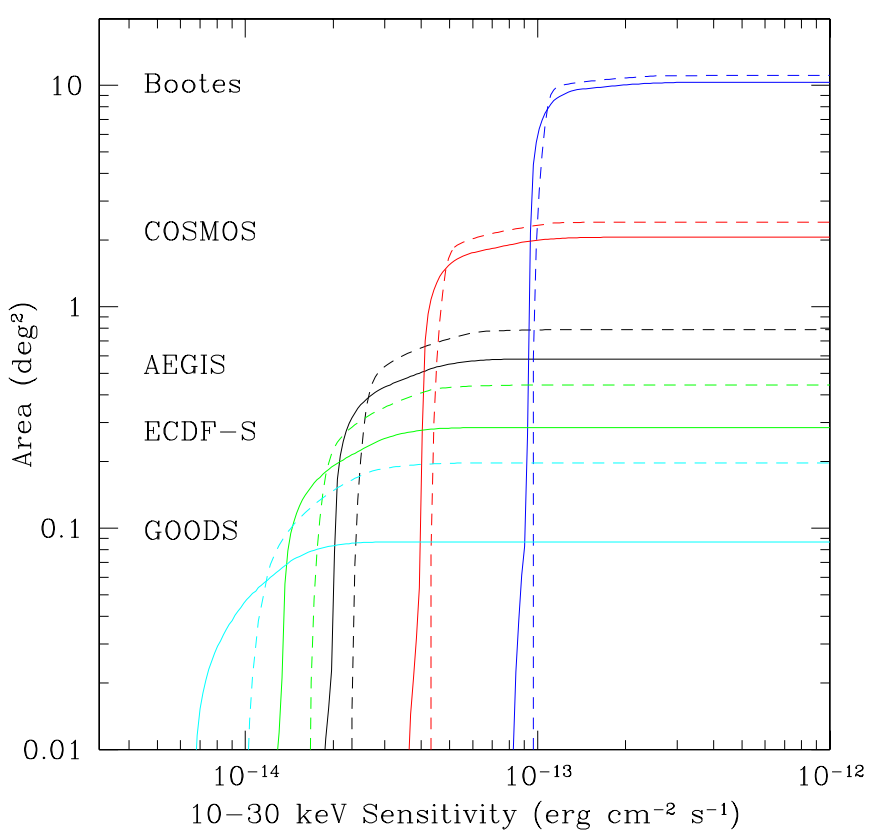

Figure 9. Area vs. 10-30 keV sensitivity for $4 \sigma$ detections of AGNs in different NuSTAR surveys with a total exposure time of $6.2 \mathrm{Ms}$ (about 6 months, assuming $50 \%$ efficiency). The solid lines are for a corner shift survey, and the dashed lines plot the results for a half shift survey. Results are shown for surveying the following regions: Boötes (blue), COSMOS (red), AEGIS (black), E-CDFS (green), and GOODS (cyan).

(A color version of this figure is available in the online journal.)

average sensitivity, and resolved fraction of the XRB - are listed in Table 1. As expected, the corner shift surveys probe slightly deeper than the half shift ones, but the latter provide more uniform coverage over the survey area, especially in the narrowfields like GOODS or ECDF-S. The numbers of AGNs predicted from both tiling strategies are nearly equal, but the wider area provided by the half shift survey increases the AGN yield by $\sim 10 \%$. Thus, we conclude that half shift tiling should be used for all $N U S T A R$ surveys.

Table 2 shows the number of AGN detections predicted by each of the five XRB models for each of the five half shift NUSTAR surveys. The significant decrease in effective area with energy is manifested in the numbers of detectable AGN: the majority of sources will be detected in the 6-10 keV band with the $30-60 \mathrm{keV}$ band yielding very few objects and basically no Compton thick objects. However, 100 AGNs will be detected in the $10-30 \mathrm{keV}$ including (depending on the model) $\sim 10-20$ Compton thick sources. Moreover, these objects will be detectable up to and beyond $z \sim 2$. These surveys will therefore increase the number of AGNs detected in hard X-rays by over an order of magnitude, and will be able to estimate the evolution of Compton thick AGNs above $z \sim 0$. In addition, good spectral measurements of absorbing column density, reflection strength, and high energy cutoff will be determined for a large number of AGNs over a range of luminosity and $z$. Such measurements were only previously available for a small number of nearby objects (Beckmann et al. 2009; Molina et al. 2009). Interestingly, because of the higher effective area and the negative $K$-correction, most Compton thick AGNs will also be detected in the $6-10 \mathrm{keV}$ band unless the exposures are very shallow (e.g., see the numbers for Boötes field in Table 2). As the NuSTAR effective area peaks at $\sim 10 \mathrm{keV}$, some fraction of the low- $z$ heavily obscured AGNs will not be detected by the deep Chandra surveys (see Figure 6). 
Table 1

NuSTAR Survey Parameters

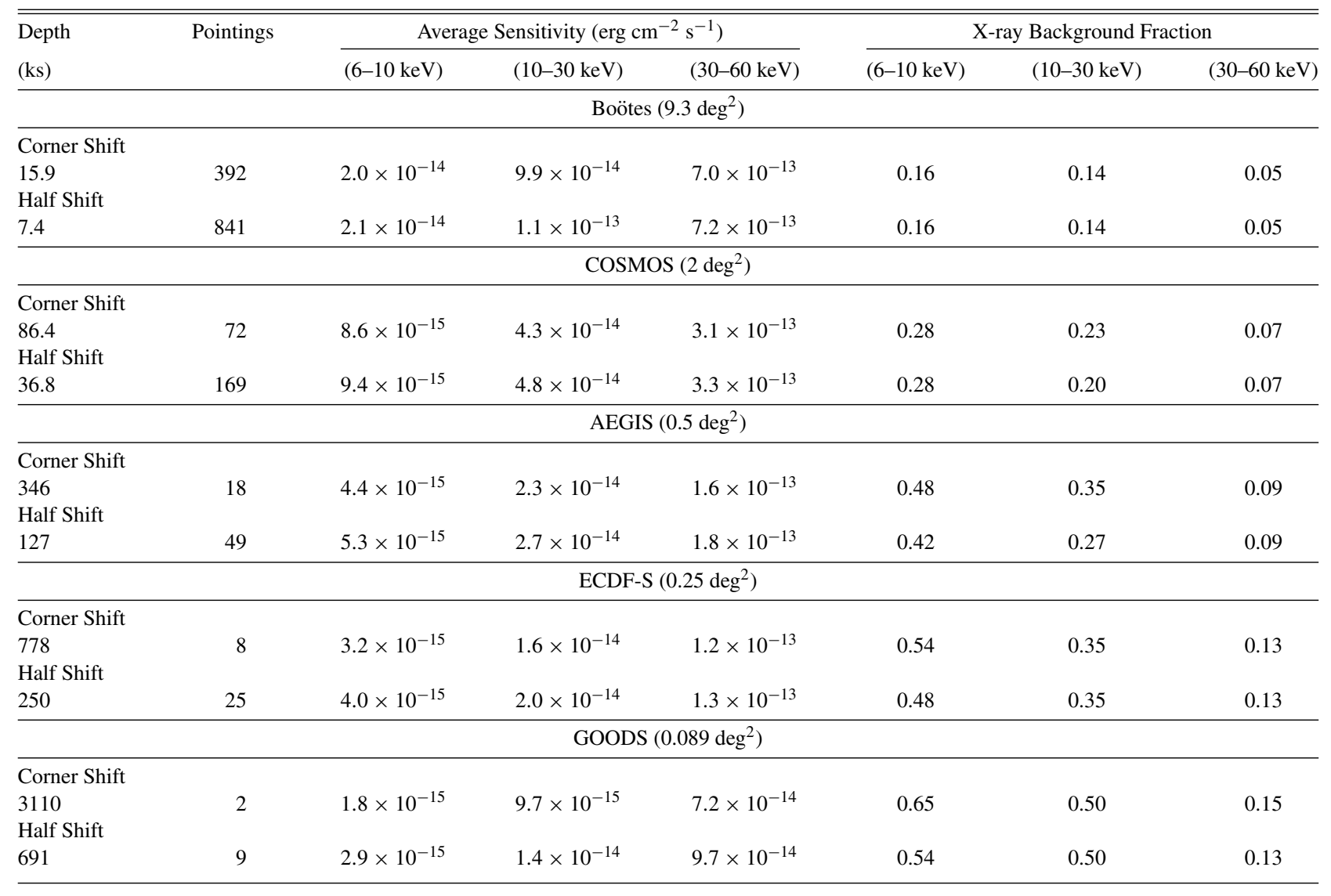

Notes. The total exposure time for each survey is $6.2 \mathrm{Ms}$, constituting a 6 month survey (assuming $50 \%$ efficiency). The average sensitivity is the flux limit of the survey at 50\% coverage (i.e., half the total area). The fraction of the X-ray background at that sensitivity is estimated using the Ueda et al. (2003) model (see Section 2.4).

The choice of NUSTAR surveys must be driven by the science goals: discovering the sources that contribute to the peak of the XRB and determining the evolution of highly obscured sources. The predicted number of Compton thick detections is roughly constant for all the surveys (except for the narrowest GOODS-like field), with the redshift distribution shifting to higher redshift as the field narrows. This fact allows some flexibility in designing a survey; for example, a $6.2 \mathrm{Ms}$ survey with an area $\sim 2 \mathrm{deg}^{2}$ will detect 15-20 Compton thick AGNs with redshifts $z \lesssim 2$. Such a survey will also resolve $\sim 30 \%$ of the XRB in $10-30 \mathrm{keV}$ band. The variation in predicted numbers of Compton thick objects implies that a simple counting experiment that can push to $z \sim 1$ may provide important constraints on the models. A very deep field such as the ECDF-S will also be of value, as it will probe a different part of the $z$-luminosity plane, and therefore would increase the statistics of the high- $z$, highly obscured population, and improve the resolved fraction of the XRB at high energies by detecting a larger number of lower luminosity AGNs at high $z$.

Finally, recall that the numbers discussed here assume that 6.2 Ms are set aside for extragalactic surveys in the baseline NuSTAR two-year mission. However, the spacecraft has no consumables and is expected to have an orbital lifetime greater than five years (Harrison et al. 2010). An extended NuSTAR mission will be able to spend several months per year building up and extending the surveys performed during the baseline mission. Thus, NUSTAR may end its mission with extragalactic surveys that have exposure times 2 to 3 times larger than the $6.2 \mathrm{Ms}$ assumed here, with the numbers of detected AGNs correspondingly larger.

\section{CONCLUSIONS}

The next few years will see the launch of the first generation of hard X-ray focusing telescopes. By focusing X-rays with energies $\gtrsim 10 \mathrm{keV}$ these missions will increase by orders of magnitude the sensitivity of observations in this energy range. This capability will be vitally important to study the growth of accreting black holes, as much of this accretion is expected to be obscured by significant columns of gas and dust. This paper presents predictions for AGN number counts in three hard X-ray bands (6-10 keV, 10-30 keV, and 30-60 keV) to understand and quantify the potential of extragalactic surveys performed by these future missions. ${ }^{15}$ The calculations pay particular attention to the expected number of Compton thick AGNs, as these heavily embedded objects may be physically connected to rapid galaxy growth and are missed by previous observations. We present predictions for five different models of the XRB and Compton thick evolution. Specifically, results are presented for three different measurements of the AGN HXLF which differ significantly in the predicted evolution of high- $z$ low-luminosity AGNs. A fourth model assumes that the

\footnotetext{
15 The results can easily be extended to other energy and flux ranges by contacting the authors.
} 
Table 2

AGN Detections for Half Shift NuSTAR Surveys

\begin{tabular}{|c|c|c|c|c|c|c|c|}
\hline \multirow[t]{2}{*}{ Model } & \multirow[t]{2}{*}{ Redshift Range } & \multicolumn{3}{|c|}{ All AGNs } & \multicolumn{3}{|c|}{ Compton Thick AGNs } \\
\hline & & $(6-10 \mathrm{keV})$ & $(10-30 \mathrm{keV})$ & $(30-60 \mathrm{keV})$ & $(6-10 \mathrm{keV})$ & $(10-30 \mathrm{keV})$ & $(30-60 \mathrm{keV})$ \\
\hline \multicolumn{8}{|c|}{ Boötes $\left(9.3 \mathrm{deg}^{2}\right)$} \\
\hline \multirow[t]{5}{*}{ Ueda et al. (2003) } & $0<z<5$ & 284 & 110 & 3 & 6 & 9 & 0 \\
\hline & $0<z<0.5$ & 113 & 65 & 3 & 3 & 7 & 0 \\
\hline & $0.5<z<1$ & 87 & 24 & 0 & 1 & 1 & 0 \\
\hline & $1<z<2$ & 52 & 10 & 0 & 2 & 1 & 0 \\
\hline & $2<z<5$ & 8 & 1 & 0 & 0 & 0 & 0 \\
\hline \multirow[t]{5}{*}{ La Franca et al. (2005) } & $0<z<5$ & 280 & 99 & 2 & 5 & 7 & 0 \\
\hline & $0<z<0.5$ & 80 & 47 & 2 & 2 & 5 & 0 \\
\hline & $0.5<z<1$ & 89 & 25 & 0 & 1 & 1 & 0 \\
\hline & $1<z<2$ & 63 & 11 & 0 & 1 & 0 & 0 \\
\hline & $2<z<5$ & 17 & 2 & 0 & 1 & 0 & 0 \\
\hline \multirow[t]{5}{*}{ Aird et al. (2010) } & $0<z<5$ & 338 & 123 & 1 & 10 & 15 & 0 \\
\hline & $0<z<0.5$ & 91 & 56 & 1 & 3 & 10 & 0 \\
\hline & $0.5<z<1$ & 155 & 47 & 0 & 5 & 4 & 0 \\
\hline & $1<z<2$ & 66 & 10 & 0 & 3 & 1 & 0 \\
\hline & $2<z<5$ & 2 & 0 & 0 & 0 & 0 & 0 \\
\hline \multirow[t]{5}{*}{ DB10 } & $0<z<5$ & 296 & 126 & 3 & 17 & 19 & 1 \\
\hline & $0<z<0.5$ & 142 & 83 & 3 & 5 & 11 & 1 \\
\hline & $0.5<z<1$ & 92 & 31 & 0 & 7 & 6 & 0 \\
\hline & $1<z<2$ & 54 & 11 & 0 & 4 & 1 & 0 \\
\hline & $2<z<5$ & 8 & 1 & 0 & 1 & 0 & 0 \\
\hline \multirow[t]{5}{*}{ Treister et al. (2009) } & $0<z<5$ & 250 & 107 & 3 & 1 & 4 & 0 \\
\hline & $0<z<0.5$ & 102 & 66 & 3 & 0 & 3 & 0 \\
\hline & $0.5<z<1$ & 78 & 28 & 0 & 0 & 1 & 0 \\
\hline & $1<z<2$ & 58 & 12 & 0 & 0 & 0 & 0 \\
\hline & $2<z<5$ & 11 & 0 & 0 & 0 & 0 & 0 \\
\hline \multicolumn{8}{|c|}{ COSMOS $\left(2 \mathrm{deg}^{2}\right)$} \\
\hline \multirow[t]{5}{*}{ Ueda et al. (2003) } & $0<z<5$ & 217 & 79 & 2 & 6 & 7 & 0 \\
\hline & $0<z<0.5$ & 59 & 36 & 2 & 2 & 4 & 0 \\
\hline & $0.5<z<1$ & 75 & 24 & 0 & 2 & 2 & 0 \\
\hline & $1<z<2$ & 60 & 12 & 0 & 2 & 1 & 0 \\
\hline & $2<z<5$ & 10 & 1 & 0 & 1 & 0 & 0 \\
\hline \multirow[t]{5}{*}{ La Franca et al. (2005) } & $0<z<5$ & 212 & 74 & 2 & 6 & 6 & 0 \\
\hline & $0<z<0.5$ & 43 & 26 & 1 & 1 & 3 & 0 \\
\hline & $0.5<z<1$ & 70 & 24 & 0 & 2 & 2 & 0 \\
\hline & $1<z<2$ & 68 & 14 & 0 & 2 & 1 & 0 \\
\hline & $2<z<5$ & 15 & 3 & 0 & 1 & 0 & 0 \\
\hline \multirow[t]{5}{*}{ Aird et al. (2010) } & $0<z<5$ & 223 & 99 & 1 & 16 & 16 & 0 \\
\hline & $0<z<0.5$ & 44 & 32 & 1 & 3 & 8 & 0 \\
\hline & $0.5<z<1$ & 97 & 45 & 0 & 8 & 7 & 0 \\
\hline & $1<z<2$ & 68 & 16 & 0 & 5 & 2 & 0 \\
\hline & $2<z<5$ & 4 & 0 & 0 & 0 & 0 & 0 \\
\hline \multirow[t]{5}{*}{ DB10 } & $0<z<5$ & 230 & 91 & 2 & 19 & 14 & 0 \\
\hline & $0<z<0.5$ & 74 & 46 & 2 & 3 & 6 & 0 \\
\hline & $0.5<z<1$ & 79 & 30 & 0 & 6 & 6 & 0 \\
\hline & $1<z<2$ & 67 & 14 & 0 & 9 & 3 & 0 \\
\hline & $2<z<5$ & 10 & 1 & 0 & 1 & 0 & 0 \\
\hline Treister et al. (2009) & $0<z<5$ & 191 & 77 & 2 & 1 & 4 & 0 \\
\hline & $0<z<0.5$ & 51 & 35 & 2 & 0 & 2 & 0 \\
\hline & $0.5<z<1$ & 65 & 26 & 0 & 0 & 1 & 0 \\
\hline & $1<z<2$ & 65 & 14 & 0 & 1 & 0 & 0 \\
\hline & $2<z<5$ & 11 & 1 & 0 & 0 & 0 & 0 \\
\hline & & & AEGIS $(0.5 \mathrm{~d}$ & & & & \\
\hline Ueda et al. (2003) & $0<z<5$ & 171 & 62 & 2 & 7 & 6 & 0 \\
\hline & $0<z<0.5$ & 34 & 22 & 1 & 1 & 3 & 0 \\
\hline & $0.5<z<1$ & 57 & 22 & 0 & 2 & 2 & 0 \\
\hline & $1<z<2$ & 61 & 13 & 0 & 3 & 1 & 0 \\
\hline & $2<z<5$ & 11 & 2 & 0 & 1 & 0 & 0 \\
\hline & & & 11 & & & & \\
\hline
\end{tabular}


Table 2

(Continued)

\begin{tabular}{|c|c|c|c|c|c|c|c|}
\hline \multirow[t]{2}{*}{ Model } & \multirow[t]{2}{*}{ Redshift Range } & \multicolumn{3}{|c|}{ All AGNs } & \multicolumn{3}{|c|}{ Compton Thick AGNs } \\
\hline & & $(6-10 \mathrm{keV})$ & $(10-30 \mathrm{keV})$ & $(30-60 \mathrm{keV})$ & $(6-10 \mathrm{keV})$ & $(10-30 \mathrm{keV})$ & $(30-60 \mathrm{keV})$ \\
\hline \multirow[t]{5}{*}{ La Franca et al. (2005) } & $0<z<5$ & 156 & 59 & 1 & 6 & 5 & 0 \\
\hline & $0<z<0.5$ & 26 & 16 & 1 & 1 & 2 & 0 \\
\hline & $0.5<z<1$ & 51 & 20 & 0 & 2 & 2 & 0 \\
\hline & $1<z<2$ & 58 & 15 & 0 & 3 & 1 & 0 \\
\hline & $2<z<5$ & 12 & 3 & 0 & 1 & 0 & 0 \\
\hline \multirow[t]{5}{*}{ Aird et al. (2010) } & $0<z<5$ & 144 & 71 & 1 & 17 & 15 & 0 \\
\hline & $0<z<0.5$ & 24 & 18 & 1 & 3 & 5 & 0 \\
\hline & $0.5<z<1$ & 58 & 32 & 0 & 7 & 7 & 0 \\
\hline & $1<z<2$ & 52 & 17 & 0 & 7 & 3 & 0 \\
\hline & $2<z<5$ & 5 & 0 & 0 & 0 & 0 & 0 \\
\hline \multirow[t]{5}{*}{ DB10 } & $0<z<5$ & 184 & 71 & 2 & 19 & 11 & 0 \\
\hline & $0<z<0.5$ & 43 & 28 & 2 & 2 & 3 & 0 \\
\hline & $0.5<z<1$ & 60 & 25 & 0 & 4 & 4 & 0 \\
\hline & $1<z<2$ & 68 & 16 & 0 & 10 & 4 & 0 \\
\hline & $2<z<5$ & 13 & 2 & 0 & 2 & 0 & 0 \\
\hline \multirow[t]{5}{*}{ Treister et al. (2009) } & $0<z<5$ & 151 & 59 & 2 & 1 & 3 & 0 \\
\hline & $0<z<0.5$ & 29 & 21 & 1 & 0 & 1 & 0 \\
\hline & $0.5<z<1$ & 49 & 22 & 0 & 0 & 1 & 0 \\
\hline & $1<z<2$ & 60 & 15 & 0 & 0 & 1 & 0 \\
\hline & $2<z<5$ & 13 & 1 & 0 & 0 & 0 & 0 \\
\hline \multicolumn{8}{|c|}{ ECDF-S $\left(0.25 \mathrm{deg}^{2}\right)$} \\
\hline \multirow[t]{5}{*}{ Ueda et al. (2003) } & $0<z<5$ & 145 & 54 & 1 & 7 & 5 & 0 \\
\hline & $0<z<0.5$ & 26 & 17 & 1 & 1 & 2 & 0 \\
\hline & $0.5<z<1$ & 48 & 20 & 0 & 2 & 2 & 0 \\
\hline & $1<z<2$ & 54 & 13 & 0 & 3 & 1 & 0 \\
\hline & $2<z<5$ & 12 & 2 & 0 & 1 & 0 & 0 \\
\hline \multirow[t]{5}{*}{ La Franca et al. (2005) } & $0<z<5$ & 129 & 51 & 1 & 6 & 5 & 0 \\
\hline & $0<z<0.5$ & 20 & 13 & 1 & 1 & 2 & 0 \\
\hline & $0.5<z<1$ & 42 & 18 & 0 & 2 & 2 & 0 \\
\hline & $1<z<2$ & 49 & 15 & 0 & 3 & 1 & 0 \\
\hline & $2<z<5$ & 11 & 2 & 0 & 1 & 0 & 0 \\
\hline \multirow[t]{5}{*}{ Aird et al. (2010) } & $0<z<5$ & 111 & 58 & 1 & 16 & 13 & 0 \\
\hline & $0<z<0.5$ & 17 & 14 & 1 & 2 & 4 & 0 \\
\hline & $0.5<z<1$ & 43 & 26 & 0 & 6 & 6 & 0 \\
\hline & $1<z<2$ & 43 & 16 & 0 & 7 & 3 & 0 \\
\hline & $2<z<5$ & 5 & 1 & 0 & 1 & 0 & 0 \\
\hline \multirow[t]{5}{*}{ DB10 } & $0<z<5$ & 156 & 62 & 2 & 17 & 10 & 0 \\
\hline & $0<z<0.5$ & 32 & 21 & 1 & 1 & 3 & 0 \\
\hline & $0.5<z<1$ & 50 & 22 & 0 & 4 & 3 & 0 \\
\hline & $1<z<2$ & 61 & 17 & 0 & 10 & 4 & 0 \\
\hline & $2<z<5$ & 14 & 2 & 0 & 3 & 0 & 0 \\
\hline \multirow[t]{5}{*}{ Treister et al. (2009) } & $0<z<5$ & 125 & 52 & 1 & 2 & 3 & 0 \\
\hline & $0<z<0.5$ & 21 & 16 & 1 & 0 & 1 & 0 \\
\hline & $0.5<z<1$ & 40 & 19 & 0 & 0 & 1 & 0 \\
\hline & $1<z<2$ & 51 & 15 & 0 & 1 & 1 & 0 \\
\hline & $2<z<5$ & 13 & 2 & 0 & 0 & 0 & 0 \\
\hline \multicolumn{8}{|c|}{ GOODS $\left(0.089 \mathrm{deg}^{2}\right)$} \\
\hline \multirow[t]{5}{*}{ Ueda et al. (2003) } & $0<z<5$ & 106 & 45 & 1 & 6 & 4 & 0 \\
\hline & $0<z<0.5$ & 17 & 11 & 1 & 1 & 2 & 0 \\
\hline & $0.5<z<1$ & 34 & 16 & 0 & 2 & 2 & 0 \\
\hline & $1<z<2$ & 41 & 14 & 0 & 3 & 1 & 0 \\
\hline & $2<z<5$ & 11 & 2 & 0 & 1 & 0 & 0 \\
\hline La Franca et al. (2005) & $0<z<5$ & 94 & 41 & 1 & 5 & 4 & 0 \\
\hline & $0<z<0.5$ & 13 & 9 & 1 & 1 & 1 & 0 \\
\hline & $0.5<z<1$ & 31 & 14 & 0 & 1 & 1 & 0 \\
\hline & $1<z<2$ & 36 & 13 & 0 & 3 & 1 & 0 \\
\hline & $2<z<5$ & 9 & 2 & 0 & 1 & 0 & 0 \\
\hline
\end{tabular}


Table 2

(Continued)

\begin{tabular}{|c|c|c|c|c|c|c|c|}
\hline \multirow[t]{2}{*}{ Model } & \multirow[t]{2}{*}{ Redshift Range } & \multicolumn{3}{|c|}{ All AGNs } & \multicolumn{3}{|c|}{ Compton Thick AGNs } \\
\hline & & $(6-10 \mathrm{keV})$ & $(10-30 \mathrm{keV})$ & $(30-60 \mathrm{keV})$ & $(6-10 \mathrm{keV})$ & $(10-30 \mathrm{keV})$ & $(30-60 \mathrm{keV})$ \\
\hline \multirow[t]{5}{*}{ Aird et al. (2010) } & $0<z<5$ & 75 & 41 & 1 & 13 & 10 & 0 \\
\hline & $0<z<0.5$ & 10 & 9 & 1 & 2 & 3 & 0 \\
\hline & $0.5<z<1$ & 27 & 17 & 0 & 5 & 5 & 0 \\
\hline & $1<z<2$ & 30 & 13 & 0 & 6 & 3 & 0 \\
\hline & $2<z<5$ & 5 & 1 & 0 & 1 & 0 & 0 \\
\hline \multirow[t]{5}{*}{ DB10 } & $0<z<5$ & 115 & 51 & 1 & 15 & 8 & 0 \\
\hline & $0<z<0.5$ & 21 & 14 & 1 & 1 & 2 & 0 \\
\hline & $0.5<z<1$ & 35 & 17 & 0 & 2 & 2 & 0 \\
\hline & $1<z<2$ & 46 & 17 & 0 & 8 & 4 & 0 \\
\hline & $2<z<5$ & 13 & 2 & 0 & 3 & 0 & 0 \\
\hline \multirow[t]{4}{*}{ Treister et al. (2009) } & $0<z<5$ & 87 & 42 & 1 & 2 & 2 & 0 \\
\hline & $0.5<z<1$ & 28 & 15 & 0 & 0 & 1 & 0 \\
\hline & $1<z<2$ & 36 & 14 & 0 & 1 & 1 & 0 \\
\hline & $2<z<5$ & 10 & 2 & 0 & 0 & 0 & 0 \\
\hline
\end{tabular}

Notes. These are $4 \sigma$ detections for the half shift surveys. Numbers for other tilings/surveys are available from the authors. The models are described in Section 2.4. The three fixed Compton thick fraction models utilize the HXLFs of Ueda et al. $\left(2003 ; f_{\mathrm{CT}}=0.3\right)$, La Franca et al. $\left(2005 ; f_{\mathrm{CT}}=0.4\right)$, and Aird et al. $\left(2010 ; f_{\mathrm{CT}}=0.5\right)$. The DB10 model is the Draper \& Ballantyne (2010) model of Compton thick evolution and makes use of the Ueda et al. (2003) HXLF. Finally, the most conservative view of the Compton thick population is based on the model of Treister et al. (2009) and also uses the Ueda et al. (2003) HXLF. Numbers have been rounded, so the sum of the individual redshift ranges may not always equal the value in the $0<z<5$ row.

Compton thick fraction is a function of the AGN Eddington ratio. The final model presents the most conservative view of the Compton thick population. These five models predict numbers of Compton thick AGNs that differ up to a factor of ten, therefore any measurement of the Compton thick number counts beyond $z=0$ will highly constrain the fraction and evolution of Compton thick AGNs.

Most hard X-ray detected AGNs will be Chandra or XMMNewton sources and have host galaxies with $R<23$; therefore, sources which are well separated from the background should be easily identified and have counterparts in multiwavelength ancillary data. Combining the hard and soft X-ray data will also allow the $N_{\mathrm{H}}$ estimates that are necessary to identify Compton thick AGNs. Other Compton thick candidates will be identified by following up sources that are undetected in the Chandra or $X M M$-Newton data. In order to make use of these data, hard $\mathrm{X}$-ray surveys should be performed in well-observed regions with deep and uniform multiwavelength coverage.

Specific predictions for five extragalactic surveys were performed for the NUSTAR mission, scheduled for launch in early 2012. All the surveys assumed a total available exposure time of 6.2 Ms, and a half shift tiling strategy is recommended. These deep surveys will yield hundreds of AGNs at $z \lesssim 2$, including (depending on the area covered) 10-20 Compton thick AGNs over that redshift range. Thus, the NUSTAR deep surveys will allow a detailed exploration of the nature and evolution of nuclear obscuration in galaxies. The simulations were appropriate for the two-year baseline mission of NUSTAR, but the surveys will ultimately probe much deeper during the extended NuSTAR mission.

The predictions presented here show that, assuming equal sensitivities, hard X-ray surveys can more efficiently detect AGNs and follow their evolution than lower energy X-ray observations. It is therefore hoped that future technology development will increase the sensitivity of hard X-ray instrumentation. A future highly efficient hard X-ray imaging mission, in combination with a ground based $30 \mathrm{~m}$ optical/IR telescope, JWST and ALMA, would be extraordinarily powerful in understanding galaxy and black hole growth throughout cosmic time.

The author list is alphabetical; all authors contributed equally to this work. This work was supported in part by NSF award AST 1008067 to D.R.B. J.R. was supported by a Carnegie Fellowship at the Carnegie Observatories in Pasadena. Support for the work of E.T. was provided by the National Aeronautics and Space Administration through Chandra Post-doctoral Fellowship Award Number PF8-90055 issued by the Chandra $X$-ray Observatory Center, which is operated by the Smithsonian Astrophysical Observatory for and on behalf of the National Aeronautics Space Administration under contract NAS8-03060. The authors thank D. Alexander, F. Harrison, and D. Stern for comments.

\section{APPENDIX}

\section{PREDICTED 5-10 keV COUNTS}

The hardest energy band that Chandra and XMM-Newton have measured AGN number counts is $5-10 \mathrm{keV}$ (e.g., Rosati et al. 2002; Della Ceca et al. 2004; Brunner et al. 2008). For completeness, and to help compare against the available data, Figure A1 plots predictions of the total and Compton thick AGN 5-10 keV counts for four of the models discussed in the paper, as well as the Gilli et al. (2007) model (magenta lines). The Compton thick counts from this last model are bracketed by the conservative model (not shown) and the Ueda et al. (2003) model at all fluxes. The cyan data show the measured counts obtained by the XMM-Newton survey of the Lockman Hole (Brunner et al. 2008) and the blue points plot the counts from the XMM-Newton Hard Bright Serendipitous Survey (Della Ceca et al. 2004). 


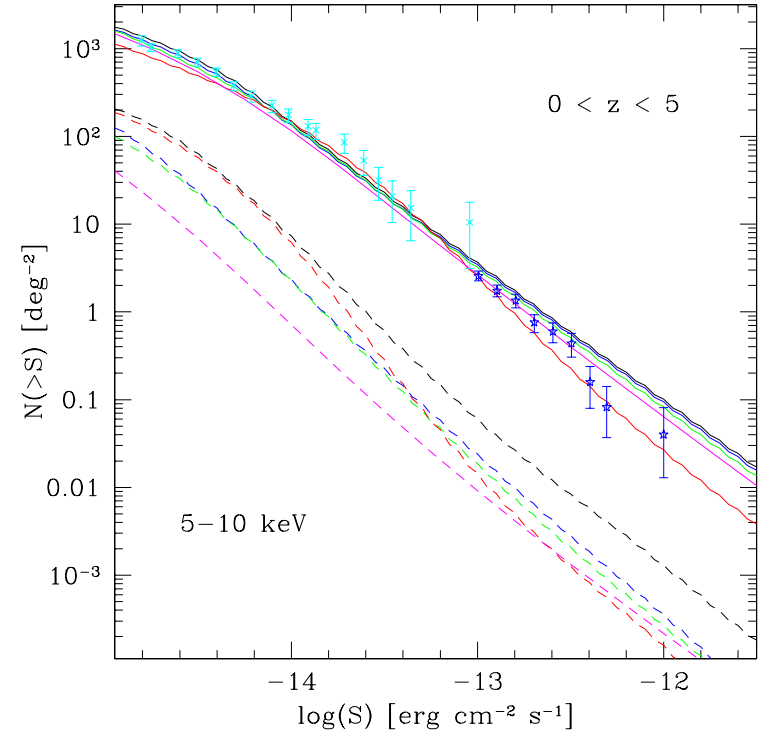

Figure A1. Predicted total (solid lines) and Compton thick (dashed lines) AGN number counts on the $5-10 \mathrm{keV}$ band. The colors denote the different XRB models as described in Figure 1. The magenta lines show the predictions of the Gilli et al. (2007) model. The cyan crosses are the measured counts from the XMM-Newton survey of the Lockman Hole (Brunner et al. 2008) and the blue stars are from the XMM-Newton Hard Bright Serendipitous Survey (Della Ceca et al. 2004).

(A color version of this figure is available in the online journal.)

\section{REFERENCES}

Agostinelli, S., et al. 2003, Nucl. Instrum. Methods Phys. Res. A., 506, 250 Aird, J., et al. 2010, MNRAS, 401, 2531

Ajello, M., et al. 2008, ApJ, 689, 666

Alexander, D. M., Brandt, W. N., Hornschemeier, A. E., Garmire, G. P.,

Schneider, D. P., Bauer, F. E., \& Griffiths, R. E. 2001, AJ, 122, 2156

Alexander, D. M., et al. 2003, AJ, 126, 539

Alexander, D. M., et al. 2008, ApJ, 687, 835

Alonso-Herrero, A., et al. 2006, ApJ, 640, 167

Ballantyne, D. R. 2010, ApJ, 708, L1

Ballantyne, D. R., Everett, J. E., \& Murray, N. 2006, ApJ, 639, 740

Barger, A. J., Cowie, L. L., Brandt, W. N., Capak, P., Garmire, G. P., Hornschemeier, A. E., Steffan, A. T., \& Wehner, E. H. 2002, AJ, 124, 1839

Barger, A. J., Cowie, L. L., Mushotzky, R. F., Yang, Y., Wang, W.-H., Steffan, A. T., \& Capak, P. 2005, AJ, 129, 578

Bassani, L., Dadina, M., Maiolino, R., Salvati, M., Risaliti, G., dell Ceca, R., Matt, G., \& Zamorani, G. 1999, ApJS, 121, 473

Beckmann, V., et al. 2009, A\&A, 505, 417

Bianchi, S., Guainazzi, M., Matt, G., \& Fonseca Banilla, N. 2007, A\&A, 467, L19

Brandt, W. N., \& Hasinger, G. 2005, ARA\&A, 43, 827

Brunner, H., Cappelluti, N., Hasinger, G., Barcons, X., Fabian, A. C., Mainieri, V., \& Szokoly, G. 2008, A\&A, 479, 283

Brusa, M., et al. 2010, ApJ, 716, 348

Burlon, D., Ajello, M., Griener, J., Comastri, A., Merloni, A., \& Gehrels, N. 2011, ApJ, 728, 58

Churazov, E., et al. 2007, A\&A, 467, 529

Comastri, A., Setti, G., Zamorani, G., \& Hasinger, G. 1995, A\&A, 296, 1

Comastri, A., et al. 2011, A\&A, 526, L9

Daddi, E., et al. 2007, ApJ, 670, 173

Davis, M., et al. 2007, ApJ, 660, L1

Della Ceca, R., et al. 2004, A\&A, 428, 383

De Luca, A., \& Molendi, S. 2004, A\&A, 419, 837

Di Matteo, T., Colberg, J., Springel, V., Hernquist, L., \& Sijacki, D. 2008, ApJ, 676,33

Donley, J. L., Rieke, G. H., Pérez-González, P. G., \& Barro, G. 2008, ApJ, 687, 111

Draper, A. R., \& Ballantyne, D. R. 2009, ApJ, 707, 778

Draper, A. R., \& Ballantyne, D. R. 2010, ApJ, 715, L99

Dwelly, T., Page, M. J., Loaring, N. S., Mason, K. O., McHardy, I., Gunn, K., \& Sasseen, T. 2005, MNRAS, 360, 1426

Ebrero, J., et al. 2009, A\&A, 493, 55
Eckart, M. E., McGreer, I. D., Stern, D., Harrison, F. A., \& Helfand, D. J. 2010, ApJ, 708, 584

Elvis, M., et al. 2009, ApJS, 184, 158

Feruglio, C., Daddi, E., Fiore, F., Alexander, D. M., Piconcelli, E., \& Malacaria, C. 2011, ApJ, 729, L4

Fiore, F., et al. 2008, ApJ, 672, 94

Fiore, F., et al. 2009, ApJ, 693, 447

Gandhi, P., Fabian, A. C., Suebsuwong, T., Malzac, J., Miniutti, G., \& Wilman, R. J. 2007, MNRAS, 382, 1005

Gendreau, K. C., et al. 1995, PASJ, 47, L5

Georgakakis, A., Rowan-Robinson, M., Nandra, K., Digby-North, J., PérezGonzález, P. G., \& Barro, G. 2010, MNRAS, 406, 420

Gilli, R., Comastri, A., \& Hasinger, G. 2007, A\&A, 463, 79

Gilli, R., Salvati, M., \& Hasinger, G. 2001, A\&A, 366, 407

Gilli, R., et al. 2011, ApJ, 730, L28

Gruber, D. E., Matteson, J. L., Peterson, L. E., \& Jung, G. V. 1999, ApJ, 520 , 124

Haiman, Z., Ciotti, L., \& Ostriker, J. P. 2004, ApJ, 606, 763

Haiman, Z., Jimenez, R., \& Bernardi, M. 2007, ApJ, 658, 721

Harrison, F. A., et al. 2010, Proc. SPIE, 7732, 77320S

Hasinger, G. 2008, A\&A, 490, 905

Hasinger, G., et al. 2007, ApJS, 172, 29

Hopkins, A. M., \& Beacom, J. F. 2006, ApJ, 651, 142

Hopkins, P. F., \& Hernquist, L. 2009, ApJ, 698, 1550

Kushino, A., Ishisaki, Y., Morita, U., Yamasaki, N. Y., Ishida, M., Ohashi, T., \& Ueda, Y. 2002, PASJ, 54, 327

La Franca, F., et al. 2005, ApJ, 635, 864

Laird, E., et al. 2009, ApJS, 180, 102

LaMassa, S. M., Heckman, T. M., Ptak, A., Hornschemeier, A., Martins, L., Sonnentrucker, P., \& Tremonti, C. 2009, ApJ, 705, 568

Lehmer, B. D., et al. 2005, ApJS, 161, 21

Levenson, N. A., Heckman, T. M., Krolik, J. H., Weaver, K. A., \& Życki, P. T. 2006, ApJ, 648, 111

Lumb, D. H., Warwick, R. S., Page, M., \& De Luca, A. 2002, A\&A, 389, 93

Luo, B., et al. 2008, ApJS, 179, 19

Magdziarz, P., \& Zdziarski, A. A. 1995, MNRAS, 273, 837

Maiolino, R., \& Rieke, G. H. 1995, ApJ, 454, 94

Malizia, A., Bassani, L., Panessa, F., de Rosa, A., \& Bird, A. J. 2009a, MNRAS, 394, L121

Malizia, A., Bassani, L., Stephen, J. B., Di Cocco, G., Fiore, F., \& Dean, A. J. 2003, ApJ, 589, L17

Malizia, A., Stephen, J. B., Bassani, L., Bird, A. J., Panessa, F., \& Ubertini, P. 2009b, MNRAS, 399, 944

Marconi, A., Risaliti, G., Gilli, R., Hunt, L. K., Maiolino, R., \& Salvati, M. 2004, MNRAS, 351, 169

Mateos, S., et al. 2008, A\&A, 492, 51

Matt, G., Pompilio, F., \& La Franca, F. 1999, New Astron., 4, 191

Merloni, A., \& Heinz, S. 2008, MNRAS, 388, 1011

Molina, M., et al. 2009, MNRAS, 399, 1293

Moretti, A., et al. 2009, A\&A, 493, 501

Murphy, K. D., \& Yaqoob, T. 2009, MNRAS, 397, 1549

Murray, S. S., et al. 2005, ApJS, 161, 1

Mushotzky, R. F. 2004, in Supermassive Black Holes in the Distant Universe, ed. A. J. Barger (Astrophysics and Space Science Library, Vol. 308; Dordrecht: Kluwer), 53

Polletta, M. d. C., et al. 2006, ApJ, 642, 673

Revnivtsev, M., Gilfanov, M., Sunyaev, R., Jahoda, K., \& Markwardt, C. 2003, A\&A, 411, 329

Rigby, J. R., Diamond-Stanic, A. M., \& Aniano, G. 2009, ApJ, 700, 1878

Risaliti, G., Maiolino, R., \& Salvati, M. 1999, ApJ, 522, 157

Rosati, P., et al. 2002, ApJ, 566, 667

Ross, R. R., \& Fabian, A. C. 2005, MNRAS, 258, 211

Sazonov, S., Revnivtsev, M., Krivonos, R., Churazov, E., \& Runyaev, R. 2007, A\&A, 462, 57

Setti, G., \& Woltjer, L. 1989, A\&A, 224, L21

Shankar, F., Bernardi, M., \& Haiman, Z. 2009, ApJ, 694, 867

Shankar, F., Salucci, P., Granato, G. L., De Zotti, F., \& Danese, L. 2004, MNRAS, 354, 1020

Shu, X. W., Yaqoob, T., \& Wang, J. X. 2010, ApJS, 187, 581

Silverman, J. D., et al. 2008, ApJ, 679, 118

Sołtan, A. 1982, MNRAS, 200, 115

Stern, D., et al. 2002, ApJ, 568, 71

Stern, D., et al. 2005, ApJ, 631, 163

Tagliaferri, G., et al. 2010, in Proc. of Science, The Extreme Sky: Sampling the Universe above $10 \mathrm{keV}$ (Trieste: SISSA) (http://pos.sissa.it/cgi-bin/reader/ conf.cgi? confid $=96$ )

Takahashi, T., et al. 2010, Proc. SPIE, 7732, $77320 \mathrm{Z}$ 
Tozzi, P., et al. 2001, ApJ, 562, 42

Tozzi, P., et al. 2006, A\&A, 451, 457

Treister, E., Natarajan, P., Sanders, D. B., Urry, C. M., Schawinski, K., \& Kartaltepe, J. 2010, Science, 328, 600

Treister, E., \& Urry, C. M. 2005, ApJ, 630, 115

Treister, E., \& Urry, C. M. 2006, ApJ, 360, 115

Treister, E., Urry, C. M., \& Virani, S. 2009, ApJ, 696, 110

Treister, E., et al. 2004, ApJ, 616, 123

Tueller, J., Mushotzky, R. F., Barthelmy, S., Cannizzo, J. K., Gehrels, N., Markwardt, C. B., Skinner, G. K., \& Winter, L. M. 2008, ApJ, 681, 113

Türler, M., Chernyakova, M., Courvoisier, T. J.-L., Lubiński, P., Neronov, A., Produit, N., \& Walter, R. 2010, A\&A, 512, A49
Ueda, Y., Akiyama, M., Ohta, K., \& Miyaji, T. 2003, ApJ, 598, 886

Ueda, Y., et al. 2007, ApJ, 664, L79

Vecchi, A., Molendi, S., Guainazzi, M., Fiore, F., \& Parmar, A. 1999, A\&A, 349, L73

Vignali, C., \& Comastri, A. 2002, A\&A, 381, 834

Winter, L. M., Mushotzky, R. F., Reynolds, C. S., \& Tueller, J. 2009, ApJ, 690, 1322

Worsley, M. A., et al. 2005, MNRAS, 357, 1281

Yencho, B., Barger, A. J., Trouille, L., \& Winter, L. M. 2009, ApJ, 698, 380

Yu, Q., \& Lu, Y. 2008, ApJ, 689, 732

Yu, Q., \& Tremaine, S. 2002, MNRAS, 335, 965 